\title{
Review
}

Joshua Christopher, Masoud Taleb, Achyut Maity, Mario Hentschel, Harald Giessen and Nahid Talebi*

\section{Electron-driven photon sources for correlative electron-photon spectroscopy with electron microscopes}

https://doi.org/10.1515/nanoph-2020-0263

Received May 1, 2020; accepted August 31, 2020; published online September 18, 2020

\begin{abstract}
Electron beams in electron microscopes are efficient probes of optical near-fields, thanks to spectroscopy tools like electron energy-loss spectroscopy and cathodoluminescence spectroscopy. Nowadays, we can acquire multitudes of information about nanophotonic systems by applying space-resolved diffraction and timeresolved spectroscopy techniques. In addition, moving electrons interacting with metallic materials and optical gratings appear as coherent sources of radiation. A swift electron traversing metallic nanostructures induces polarization density waves in the form of electronic collective excitations, i.e., the so-called plasmon polariton. Propagating plasmon polariton waves normally do not contribute to the radiation; nevertheless, they diffract from natural and engineered defects and cause radiation. Additionally, electrons can emit coherent light waves due to transition radiation, diffraction radiation, and SmithPurcell radiation. Some of the mechanisms of radiation from electron beams have so far been employed for designing tunable radiation sources, particularly in those energy ranges not easily accessible by the state-of-the-art laser technology, such as the $\mathrm{THz}$ regime. Here, we review various approaches for the design of coherent electrondriven photon sources. In particular, we introduce the
\end{abstract}

*Corresponding author: Nahid Talebi, Institute for Experimental and Applied Physics, Christian Albrecht University, 24107 Kiel, Germany; and Max Planck Institute for Solid State Physics, 70569 Stuttgart, Germany, E-mail: talebi@physik.uni-kiel.de. https://orcid.org/00000002-3861-1005

Joshua Christopher and Masoud Taleb, Institute for Experimental and Applied Physics, Christian Albrecht University, 24107 Kiel, Germany Achyut Maity, Max Planck Institute for Solid State Physics, 70569 Stuttgart, Germany

Mario Hentschel and Harald Giessen, 4th Physics Institute and Research Center SCoPE, University of Stuttgart, Pfaffenwaldring 57, 70550 Stuttgart, Germany theory and nanofabrication techniques and discuss the possibilities for designing and realizing electron-driven photon sources for on-demand radiation beam shaping in an ultrabroadband spectral range to be able to realize ultrafast few-photon sources. We also discuss our recent attempts for generating structured light from precisely fabricated nanostructures. Our outlook for the realization of a correlative electron-photon microscope/spectroscope, which utilizes the above-mentioned radiation sources, is also described.

Keywords: cathodoluminescence; coherence; electrondriven photon source; mechanisms of radiation.

\section{Introduction}

Probing materials with electron beams to investigate their underlying physical and chemical properties has a long tradition. Indeed, cathodoluminescence (CL), which is the ultraviolet/infrared/visible radiation from a material as an electron interacts with it, started with the development of cathode tubes [1, 2]. Arguably, for optical and structural characterization of nanomaterials, CL and electron energyloss spectroscopy (EELS) provide the best energy, space, and time resolution. Unlike monochromatic laser light, the electromagnetic field accompanying a moving electron can excite various optical modes when interacting with luminiscent materials. Similar to the effect of an optical pulse on a target material, CL and EELS excitation mechanisms involve polarizing the target material on impact by the time-varying electric fields produced by the moving electron. The coupling of propagating light with electrons confined in atoms, molecules, or bulk material is almost a natural phenomenon. However, the reverse, which is the coupling of propagating free electrons with localized optical fields, happens only in electron microscopes. The basics of this interaction are succinctly captured in the Kapitza-Dirac effect introduced a long time ago [3] and recently experimentally detected $[4,5]$ and further 
generalized to include arbitrary light shapes [6]. To explore the possibilities of retrieving the sample dynamics using electron microscopes, an in-depth understanding of the interaction between ultrafast electron wave packet, intense ultrafast laser pulse, and nanostructure is crucial [7-10]. Recent efforts toward retrieving the dynamics is captured in the so-called field of photoinduced near-field electron microscopy [11].

The spatial extents of the electric field around the electron's trajectory are elliptical and decay evanescently, particularly in aloof excitation. The incident and induced electromagnetic field on the target material by the moving electron has well-defined phase relation, in addition to ultrafast, broadband, nanometric precision properties continuously being unraveled via advances in this spectroscopy technique. Also, coherent CL radiation can be tailored utilizing electron-driven photon sources (EDPHSs) to facilitate time-resolved CL without the need to manipulate electron microscopes with external laser excitation. EELS is usually compared with optical extinction and CL spectroscopy with scattering measurements. Extinction comprises both the scattered and absorbed electromagnetic field on the impact of the electron with the target material [12]. When a relativistic electron beam impacts a luminescent material, incoherent or coherent radiation can be generated [13]. If the radiation has constant phase relation with the electric field of the incident electron, then the radiation is coherent, otherwise it is incoherent. There have been exciting advances in recent times concerning coherent EELS and CL spectroscopies. Via pulsed laser photoemission, ultrashort electron pulses have been created in the electron cathode, which paves the way for ultrafast electron microscopy and time-resolved CL [14, 15]. In addition, coherent CL radiation can be tailored by employing EDPHSs to facilitate ultrafast CL spectroscopies without the need to manipulate electron microscopes with external laser excitations [16]. The state of the emitted CL radiation can be fully characterized through angle- and polarization-resolved CL.

The evanescent field of a moving electron can couple with the target material leading to electromagnetic radiation such as transition radiation (TR) [17], Cherenkov radiation (CR) [18], Smith-Purcell radiation (SPR) [19], bremsstrahlung radiation (BR) [20], and a host of other excitation events. Electrons traveling parallel to a metallic diffraction grating emit SPR (Figure 1A). Whether the emission is coherent or incoherent depends on several factors such as the shape of the electron wave function, the structure, and the nature of the metallic grating [21-24]. In the case where the electron traverses a metal/dielectric interface, TR is emitted. It is generally accepted that TR occurs due to the annihilation of the image charge created by the incident electron on crossing the dielectric/metallic interface (Figure 1B). TR is usually coherent and has been employed in quantifying coherent and incoherent CL in semiconductors and metals [25]. In addition to TR, excited surface plasmon polaritons (SPPs) propagating along the surface can cause radiation when interacting with defects or a grating. These coupled transverse electromagnetic fields and charge density oscillations, which propagate along the dielectric/metallic surface, are strongly bound to the surface. This makes them suitable for applications in signal processing and broadband interconnection [26]. In the design of EDPHSs, the outcoupled radiation due to propagating SPPs on defects or gratings can be harnessed and manipulated for enhanced directional radiation for spectroscopic applications. A schematic diagram for a typical experimental setup for the generation of SPPs is shown in Figure 1C.

There have been extensive studies on the interaction of electrons with nanostructures covering a broad range in plasmonics and materials science [28-31]. However, this review emphasizes design approaches for coherent EDPHSs for spectral interferometry applications. Understanding the underlying properties of materials at the atomic scale encompasses the field of nanoscience. So far, advances in nanoscience have led to intriguing applications and the miniaturization of several technological devices. The EDPHS when incorporated into the state-of-theart electron microscopes can further enhance the spectroscopic analysis of nanomaterials. The focused TR emitted by the EDPHS and the propagating electron, both on interaction with the sample, which emits a secondary radiation can be collected by the CL detector. Also, the EDPHS can be harnessed for quantum optical experiments. We start by reviewing various possible coherent sources of radiation such as CR sources, TR sources, surface plasmon (SP)-induced radiation sources, coherent BR sources, metamaterial-based sources, SPR sources, holographic sources, and photon sieve sources. Each of these radiation sources provides coherent radiation needed for the design of EDPHSs. On the other hand, our recent attempts in fabricating various geometric photon sieve structures for light focusing are also discussed alongside their far-field angle-resolved CL maps. This review also presents insights into spectral interferometry with EDPHSs.

In Section 2 of the current review, mechanisms of radiation from electron beams interacting with materials are classified and several explored systems are provided. In chapter 3, recent developments and engineering concepts for harnessing the electron-induced radiations for possible miniaturized sources are provided. We particularly cover metamaterial sources in Section 3.1, SPR sources in Section 
A

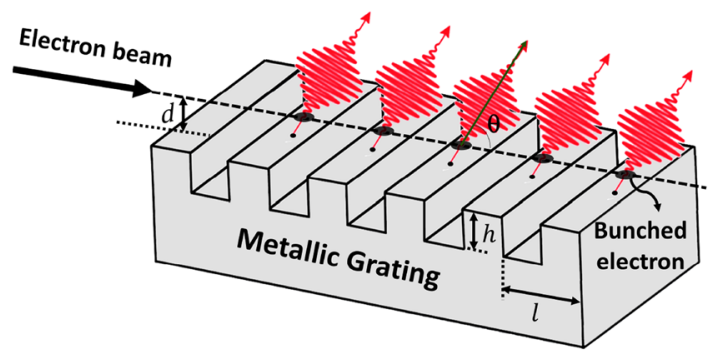

B

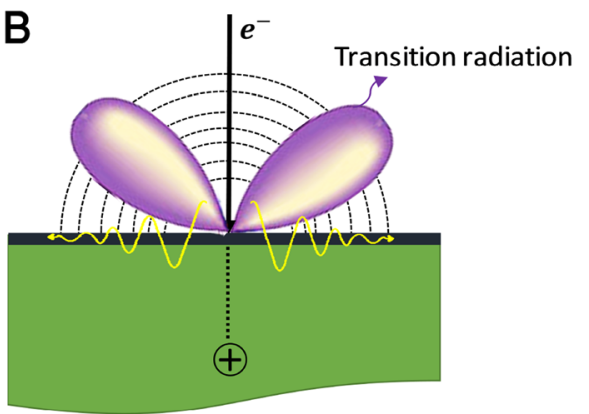

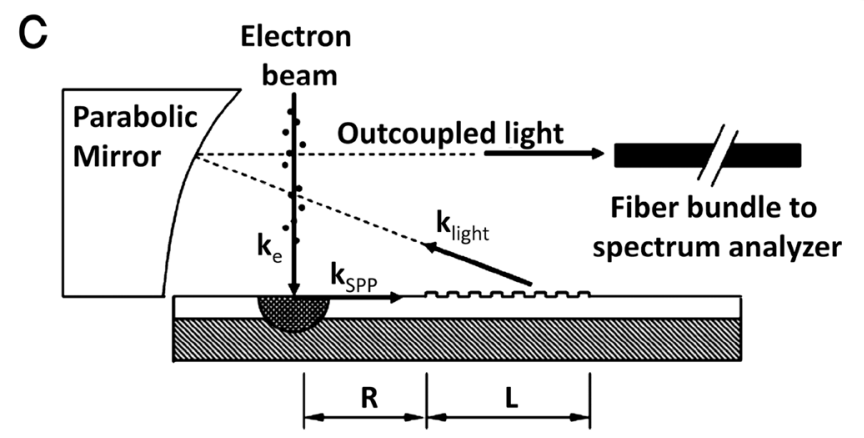

Figure 1: Schematics diagram of different mechanisms of radiations from electron beams interacting with nanoscopic and mesoscopic matter. (A) Smith-Purcell radiation as a result of an electron traveling parallel to the surface of a metallic grating. (B) Emitted transition radiation when the electron passes through the material and dielectric. (C) Experimental setup for excitation of surface plasmon polariton with wave vector $\mathbf{k}_{\mathrm{SPP}}$ by direct injection of a beam of free electrons of wave vector $\mathbf{k}_{e}$ and their decoupling as a light by grating [27].

3.2, holographic sources in chapter 3.3, and photon sieve sources in chapter 3.4. Metamaterial concept introduced to design materials with dielectric functions not obviously provided by nature is considered generally. Indeed, some holographic and photon sieve structures might be categorized under the metamaterials family as well. Nevertheless, for historical reasons, we have decided to not merge these concepts. In Section 4, an outlook for using EDPHSs for spectral interferometry with electron microscopes is provided. In Section 5, different methods used for realizing and characterizing some of the EDPHS structures are outlined.

\section{Mechanism of electron-matter interaction}

Electrons can be modeled classically as localized charge or quantum mechanically. The quantum dynamics of propagating electron-matter interaction, well captured in the celebrated quantum electrodynamics, although complicated, gives an accurate description of this interaction. When an electron interacts with matter, there are several resulting dynamics. The electron can excite various optical modes, which are usually reflected as photon emission or absorption and other phenomena. This is usually a complicated process and dependent on the kind of electron-matter interaction to be studied. This section describes basic concepts for photon generation for EDPHS design on electron interaction.

\subsection{Cherenkov radiation}

A charged particle propagating through a bulk material emits CR when its velocity exceeds the phase velocity of light in that medium $[18,32]$. Some of the earliest theoretical works describing this fact have been discussed elsewhere [33-35]. Considering a medium with a refractive index $n$ where a particle, for example, an electron, travels through with a velocity $v$, the particle velocity can become larger than that of light in that medium. This leads to the formation of the Cherenkov cone of radiation behind the particle. In the case of reversed CR in metamaterials, the emitted radiation is in the opposite direction to the propagation of the electron [36]. In particular, left-handed metamaterials shows this phenomenon [37]. The concept of reversed CR and the negative phase velocity of light have been demonstrated for an electron propagating in the bulk of a left-handed material via the calculated momentumresolved electron energy-loss (EEL) and CL spectra [38]. Scientists have made several attempts toward quantifying this radiation [39-41]. 
CR can be observed in a transmission electron microscope when an electron interacts with the sample. EELS or CL spectra show various resonance phenomena for different materials [42, 43]. The in-plane dispersion and energy-momentum isofrequency surfaces for anisotropic materials can be unraveled by direct detection of $\mathrm{CR}$ emission using angle-resolved CL spectroscopy and momentum-resolved EELS (MREELS) [44-46]. The isofrequency surfaces of planar light waves in hyperbolic materials mimic the hyperbola shape, whereas, for anisotropic dielectrics, their isofrequency surfaces are ellipsoids. Natural hyperbolic metamaterials such as gallium telluride (GaTe) [47], barium titanate $\left(\mathrm{BaTiO}_{3}\right)$ [48], silicon dioxide $\left(\mathrm{SiO}_{2}\right)$ [49], bismuth telluride $\left(\mathrm{Bi}_{2} \mathrm{Te}_{3}\right)$ [50], bismuth selenide $\left(\mathrm{Bi}_{2} \mathrm{Se}_{3}\right)$ [51, 52], and hexagonal boron nitride ( $\mathrm{h}$ $\mathrm{BN})$ [53] have been extensively studied. In particular, $\mathrm{Bi}_{2} \mathrm{Te}_{3}$ and $\mathrm{h}$-BN will be elucidated here. $\mathrm{Bi}_{2} \mathrm{Te}_{3}$ is a uniaxial material with its permittivity along the ab crystallographic plane being different from the permittivity normal to the plane. Hence, an electron moving perpendicular or parallel to the optics axis, interacting with the materials, excites various optical modes. At frequencies above $3 \mathrm{eV}, \mathrm{Bi}_{2} \mathrm{Te}_{3}$ demonstrates a type II hyperbolic behavior with $\epsilon_{\|}<0$ and $\epsilon_{\perp}>0$ (Figure 2A). Recently, high-momentum modes and hyperbolic $\mathrm{CR}$ in $\mathrm{Bi}_{2} \mathrm{Te}_{3}$ have been investigated experimentally [50]. The experimental demonstration was carried out using the MREELS technique. Figure 2B shows experimental MREELS measurements over a range of different scattering angles (momentum) for $60-\mathrm{nm} \mathrm{Bi}_{2} \mathrm{Te}_{3}$ film prepared via focused ion beam. The three angles set show a broad peak which corresponds to high-momentum modes and hyperbolic CR modes excited in the sample.

$\mathrm{H}-\mathrm{BN}$ is a hyperbolic material at the lower and upper reststrahlen band at the far-infrared range [54]. The reststrahlen band is the energy range between the longitudinal and transverse optical phonons. h-BN supports optical phonons both along the $c$-axis and within the $a b$ planes. Recently, forward and backward CR for phonon polaritons has been theoretically reported for 105-nm-thick h-BN structure [55]. CR was excited via propagating electrons at different velocities, $20 \mathrm{~nm}$ above the structure. Figure $2 \mathrm{C}$ shows the $z$ components of the electric field for propagating electrons at $0.02 \mathrm{c}$, with $c$ being the free-space light velocity. Figure 2D shows the phonon polariton spectrum for a moving electron at different velocities. The spectrum depicts two midinfrared broadband peaks in the upper and lower restrahlen band of h-BN. In the upper restrahlen band, the spectrum intensity peak depicts a redshift as electron velocity increases. However, it exhibits a blueshift in the lower restrahlen band. MREELS of vibrational modes in $\mathrm{h}$-BN with an unprecedented energy resolution has been recently demonstrated [56]. By improving further the momentum resolutions, the interaction of $\mathrm{CR}$ with phonon vibrations can be detected by the cutting-edge transmission electron microscopy instrumentation. Finally, it should be mentioned that excitations in thin films can be significantly different from those of bulk materials. In thin films made of $\mathrm{Bi}_{2} \mathrm{Se}_{3}$, surface exciton polaritons and other waveguiding modes occur and significantly alter the MREELS spectra. Similarly, in the h-BN thin films, phonon polaritons can be excited as well.

Recently, it was shown that a moving charge interacting with a hyperbolic material can lead to CR emission without any predefined value of the kinetic energy of the electron [57]. These findings can be used in the design of a tunable light source made of hyperbolic metamaterials. Also, CR finds application in the detection of biomolecules and medical imaging of radioisotopes. In the field of particle physics, CR can be used in the identification of particles by the properties of CR they emit in the medium where they propagate.

\subsection{Transition radiation}

The phenomena of TR were described by Ginzburg and Frank [58] in 1945. They considered the case of an electron incident normally on the boundary between the vacuum and a perfect conductor. They derived a formula for the energy $(W)$ radiated in a unit solid angle in the relativistic case [58]:

$$
W=\frac{e^{2} v^{2} \sin ^{2} \theta}{\pi^{2} c^{3}\left(1-\beta \cos ^{2} \theta\right)^{2}},
$$

where $e$ is electron charge, $v$ is the velocity of the electron, $\theta$ is the angle of emission of the TR, $c$ is the velocity of light in vacuum, and $\beta=\frac{v}{c}$. Thus, TR can be described as the radiation emitted when the electron transverses the boundary of two homogeneous media. One of the media is a metal while the other is a dielectric. TR is dependent on both the velocity of the electron and the optical properties of the conducting medium. TR is mutually coherent with the selffield of the electron. It is widely accepted that TR occurs as a result of the interaction of the moving electron with its image charge (Figure 3A). This involves (i) creation of the image charge in the second medium as the electron is still moving in the first medium, (ii) a sharp annihilation of the image charge when the electron crosses the interface, and (iii) screening of the electron charge when the electron has crossed the interface. The TR emission pattern 
A

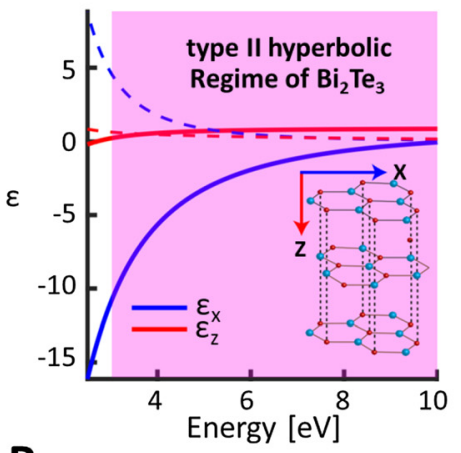

B

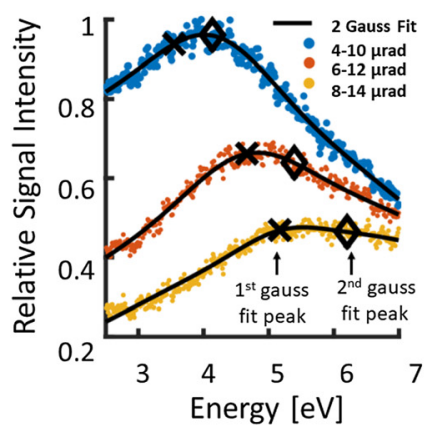

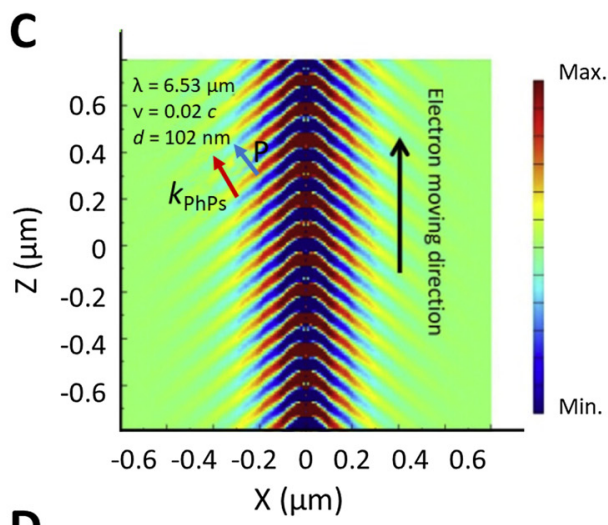

D

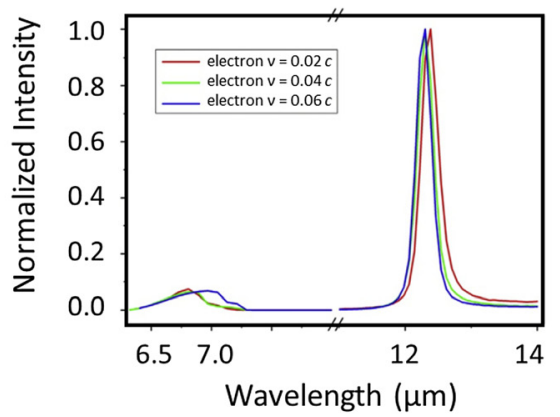

Figure 2: (A) Uniaxial dielectric permittivity of $\mathrm{Bi}_{2} \mathrm{Te}_{3}$ parallel $\left(\epsilon_{z}\right)$ and perpendicular $\left(\epsilon_{x}\right)$ to the $c$-axis showing type II hyperbolic behavior $\left(\epsilon_{x}<0, \epsilon_{z}>0\right)$ in the visible to the ultraviolet spectrum range. (B) Experimental energy loss peaks for fast electrons with a velocity of $V_{z}=0.78 \mathrm{c}$ normally incident on the sample of a $60-\mathrm{nm} \mathrm{Bi}_{2} \mathrm{Te}_{3}$ thin film with no substrate. Three different summed angular ranges of the scattered electrons are shown. The broad peak in each of the three sets consists of the high-momentum modes and the hyperbolic Cherenkov mode. A summation of two gaussian distributions is used to fit the data, and the peak positions are marked by crosses and diamonds [50]. (C) Simulated electric field $\left(E_{z}\right)$ distributions $20 \mathrm{~nm}$ above the $\mathrm{h}-\mathrm{BN}$ layer at $6.4 \mu \mathrm{m}$ wavelenght. The thickness of $h-B N$ is $d=105 \mathrm{~nm}$, and the electron velocity is $V=0.02$ c. (D) Phonon polariton radiation spectrum for a moving electron above the h-BN layer with the thickness of $105 \mathrm{~nm}$ at velocities of $V=0.02,0.04$, and 0.06 c [55]. mimics a toroidal form. However, when silicon-based semiconductor materials interact with an electron, the radiation pattern mimics a Lambertian profile (Figure 3B) [25]. Based on these observations, Brenny et al. [25] presented a method for quantifying coherent and incoherent CL for semiconductors and metals using angle-resolved CL. Their method relied on the radiation pattern of the emission from semiconductors and metals for quantifying the coherent emission. They measured the CL spectra for $\mathrm{Si}$ and other metals/semiconductors such as $\mathrm{GaAs}, \mathrm{Al}, \mathrm{Ag}, \mathrm{Au}$, and $\mathrm{Cu}$. They decomposed the CL spectra of each metal/ semiconductor into coherent and incoherent radiation. For silicon, at 30 and $5 \mathrm{keV}$ electron kinetic energies, the CL spectra showed both coherent TR and luminescent radiation (Figure 3D). The experimental results were compared with the theoretical calculation of TR, and a very good convergence was found (Figure 3E).

TR radiation has been studied both theoretically and experimentally [17, 59-61]. Ginzburg [62], who was the first to predict the TR phenomenon, carried out a theoretical approach in several ways. He treated TR as the radiation created due to a sharp change in the velocity of the electron at the interface [62] or changes in the phase velocity of light as a result of the inhomogeneity of the medium [63]. A theoretical treatment based on the nonrecoil approximation is well suited to describe this phenomenon. TR contributes to the EEL spectra, and a detailed analytic treatment of the EEL probability has been performed by Kroger [64] in 1968 and recently by De Abajo et al. [65] in more detail by considering inclined excitation toward the surface. Such a treatment has shown good agreement with experimental results if the retardation effect and boundary condition are taken into account.

Metallic and dielectric thin films support guided waves as well. The dispersion of guided waves can be unraveled by MREELS. In isotropic metallic thin films below the plasma frequency, the overall radiation contains contributions from TR and SPs. The field profile of a metallic thin film can be decomposed into symmetric and asymmetric configurations formed by the coupling of SPs at the interface. The MREELS for pure thin films displays this hybridization effect. When thin films are stacked in several layers, the phenomenon is slightly different. As the electron interacts with this structure, interference occurs within the structure due to multiple reflections of emitted light at the boundaries. Thus, the energy-loss spectra are affected significantly [60].

The radiation caused by electrons transversing an interface can either be coherent or incoherent. This can be inferred from the radiation pattern. This pattern is similar to that of a dipole positioned at the metal/dielectric interface and aligned normal to it with a slightly different 


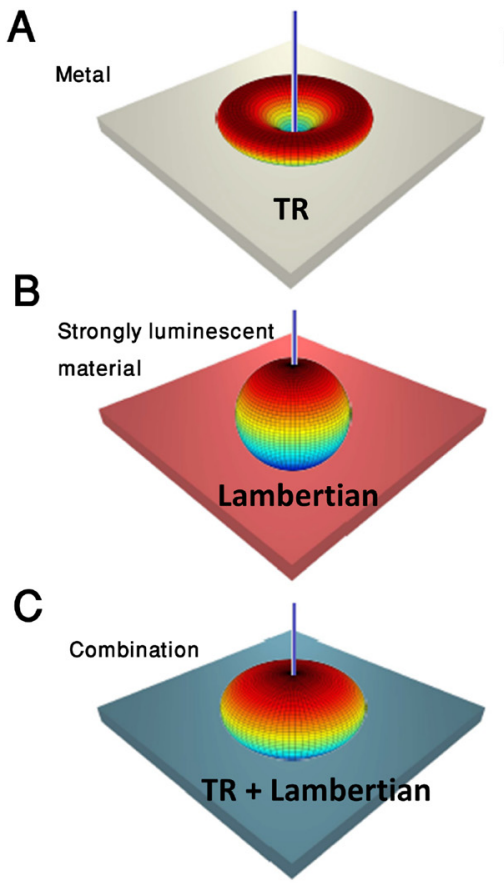

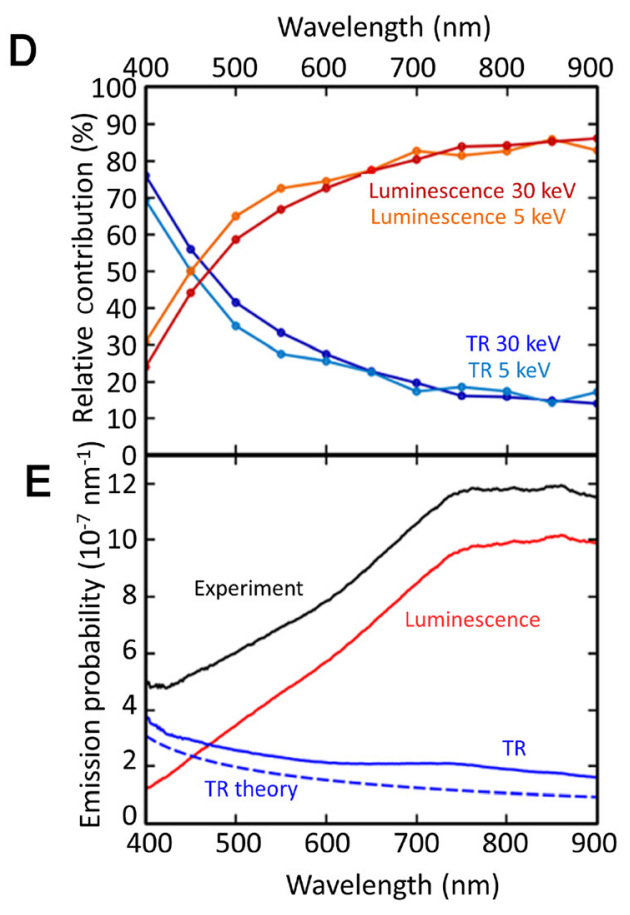

Figure 3: (A) Schematic angular emission profile for electron beam-induced radiation from a metal, which is dominated by transition radiation (TR). (B) Schematic angular emission profile for incoherent luminescence generated inside the material with a Lambertian emission profile. (C) Schematic emission profile for a combination of TR and luminescence, which is the case for $\mathrm{Si}$. (D) The relative contribution of TR and luminescence derived from fits to the silicon emission pattern for 5 and $30 \mathrm{keV}$ energy. (E) The CL spectrum (black) together with TR (blue) and incoherent luminescence (red) contribution for Si at $30 \mathrm{keV}$. The dashed blue lines show the theoretical TR spectrum for Si at $30 \mathrm{keV}$ [25]. CL, cathodoluminescence; TR, transition radiation. orientation of the maximum radiation intensity [13]. An electron transversing an Au surface can excite both TR and SPs (Figure 4A, C, and D). SPs do not contribute to the radiation unless the surface of the metal is engineered or defects are exposed. However, for anisotropic hyperbolic materials, two different transverse electric polariton modes can be excited. Moreover, the dispersion of one of those modes can be located inside the light cone, resulting in radiative surface modes [66]. Metamaterials, on the other hand, have been used for generating and manipulating TR radiation. By carefully tuning the refractive index of metamaterials, TR can be manipulated [67]. In particular, bismuth selenide $\left(\mathrm{Bi}_{2} \mathrm{Se}_{3}\right)$, a natural hyperbolic metamaterial, shows an interesting interplay between coherent and incoherent emission when an electron transverses its surface. Since the material is hyperbolic, the radiation pattern shows a mixture of dipolar-like and Lambertian emission profiles (Figure 4B-D).

\subsection{Surface plasmon-induced radiation}

The field of plasmonics which deals with the optical properties of metal structures at the nanoscale has gained significant attention in recent years. Just as phonons are quanta of mechanical vibrations, photons the quanta of light, plasmons are quanta of plasma oscillations. SPs are collective oscillations of free electrons on the surface of metals induced by the coupling of an external electromagnetic field with the free electrons in the metal $[51,68]$. SPPs are confined to the surface of the metal and strongly interact with photons [69]. Typically, the interface between a dielectric with positive real permittivity and a material with negative real permittivity such as a heavily doped semiconductor or a metal support SPs [70]. Pioneered by Ritchie [71] in 1957, when analytically treating the energy losses experienced by a moving electron interacting with metallic surfaces, electron beam probes have been continuously used to retrieve collective excitations known as surface plasmons. Ritchie's work was soon after confirmed by Powel and Swan [72, 73], and later on, even localized plasmons of several nanostructures were investigated by Batson [74] and Ugarte et al. [75], confirming the possibility of using energy-loss spectroscopy to map collective excitations. In a more recent work, Nelayah et al. [76] demonstrated the possibility of combing spectroscopy and spatial scanning to map the spatial distribution of various localized plasmon modes. The method detects plasmons as resonance peaks in the energy-loss spectra of subnanometer electron beams rastered over nanoparticles of well-defined geometrical parameters. The method was applied to a triangular silver nanoprism synthesized by light-induced aggregation of $5.5 \mathrm{~nm} \mathrm{Ag}$ nanoparticles in addition to another method [77]. Figure 5A shows the associated EEL spectra and geometry of the Ag nanoprism.

SPs can confine the electromagnetic energies at nanoscale volumes well beyond the diffraction limit of light. This confinement effect is concomitant with 

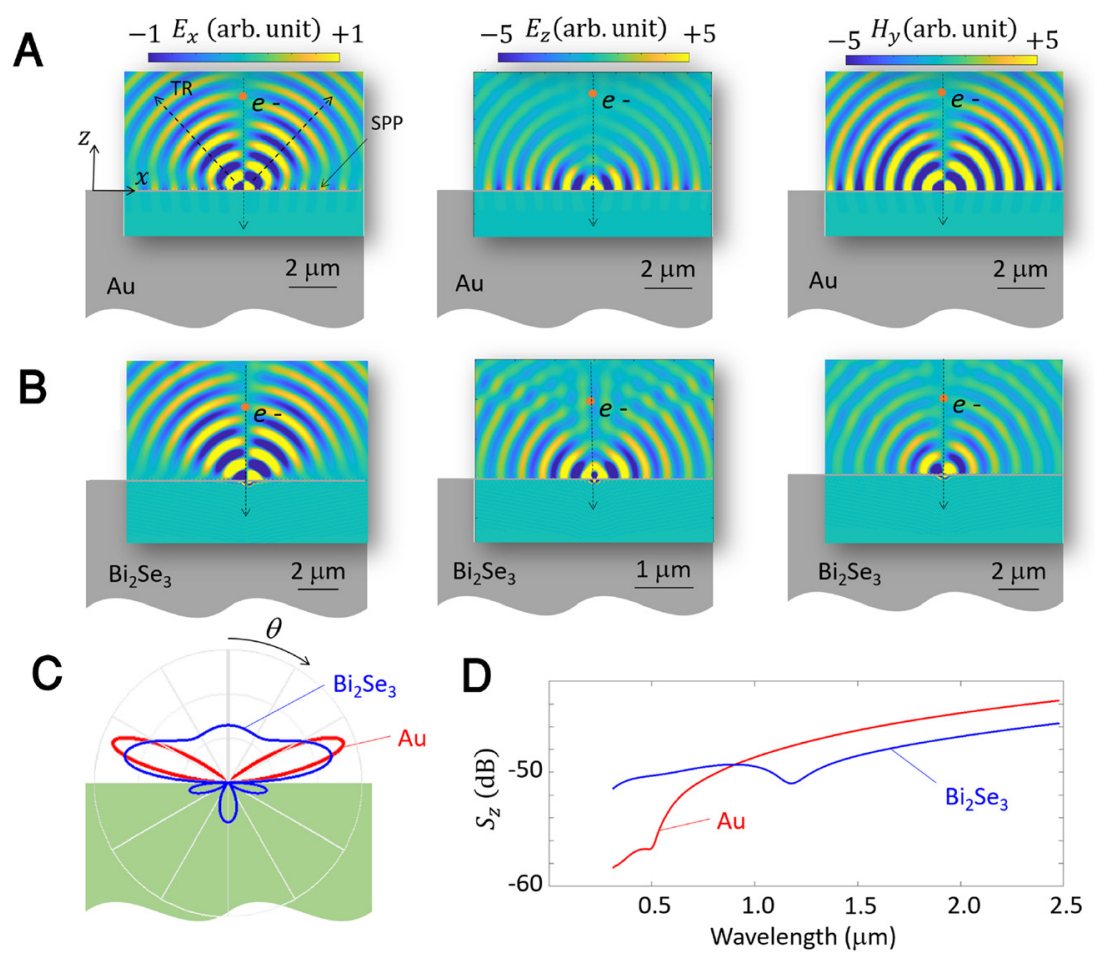

Figure 4: Calculated scattered amplitude for (A) vacuum/gold (B) vacuum $/ \mathrm{Bi}_{2} \mathrm{Se}_{3}$ interface for a moving electron transversing the surface of a material normal to the surface plane. (C) Radiation pattern of the emitted photons at an energy of $E=0.8 \mathrm{eV}$ for gold and $\mathrm{Bi}_{2} \mathrm{Se}_{3}$ surface. (D) Radiation power spectra ( $z$ component of the poynting vector $S_{z}$ ) in the positive $z$ direction for the TR from $\mathrm{Au}$ and $\mathrm{Bi}_{2} \mathrm{Se}_{3}$ surfaces [54]. TR, transition radiation. enhanced optical field intensities. Additionally, nanoscale gaps between two metallic structures can confine electromagnetic radiation due to the coupling between propagating SPs and localized particle plasmons. These gaps can be formed by depositing a metallic nanoparticle on a metallic substrate with a precise nanometer distance between them [78]. Yamamoto [79] showed that gap plasmons can be observed for a silver nanosphere deposited on a silver substrate, when the beam spot is close to the center of the spheres. The angular dependence of the emission was measured, and three peaks were revealed for the beam scan spectral and photon maps taken at the emission energies. The three peaks correspond to the quadrupole and dipole Mie resonances as well as gap mode, respectively (Figure 5B). In another related work, Sannomiya et al. [80] studied the interference between substrate TR and the field resulting from the outcoupling of SPP excitation when the silver nanosphere was deposited on a silver substrate. The interference pattern was employed in the visualization of the phase associated with the plasmonic field of metallic surface composites through nanoscopically resolved CL using a scanning transmission electron microscope [81] (Figure 5C).

Shape engineering plays a vital role in the manipulation of radiation properties generated by plasmons. By engineering the shape of the metal and varying the dielectric environment, the resonance wavelength of SPs can be tuned. Engineered surfaces such as rectangular strips, cylinders, v-grooves, and others present better opportunities for manipulating the generated plasmons. This provides room for tailored plasmon excitation for desired optical applications. It has been experimentally demonstrated that suitably engineered plasmonic resonant nanoparticle chains exhibit nanoscale localized responses to unfocused light that can be controlled by tuning the incident wavelength [82]. Also, nanoshells, nanorings, and nanorods can be used to effectively control plasmon resonances [83, 84]. Resonant coupling between silver nanoparticles fabricated using electron beam lithography and liftoff on fused silica concentrates lights into well-defined hot spots enabling ultrasmall and wavelength-sensitive detectors. The response of metallic nanostructures and nanoparticles to external electromagnetic radiation is dominated by SP generation and critically depends on the nature of the local structure and geometry. Figure 5D shows the annular dark-field scanning transmission electron microscope image of a cluster of three gold particles and the corresponding resonant modes, captured by EELS [85].

Metals such as gold, silver, aluminum, copper, gallium, and alkali metals show plasmonic properties with varying advantages and disadvantages [86-90]. Threedimensional plasmonic gold tapers have the unique ability to focus light into subwavelength volumes in a coherent, broad, and controllable manner. Plasmon-induced radiations in the visible range for three-dimensional gold tapers 


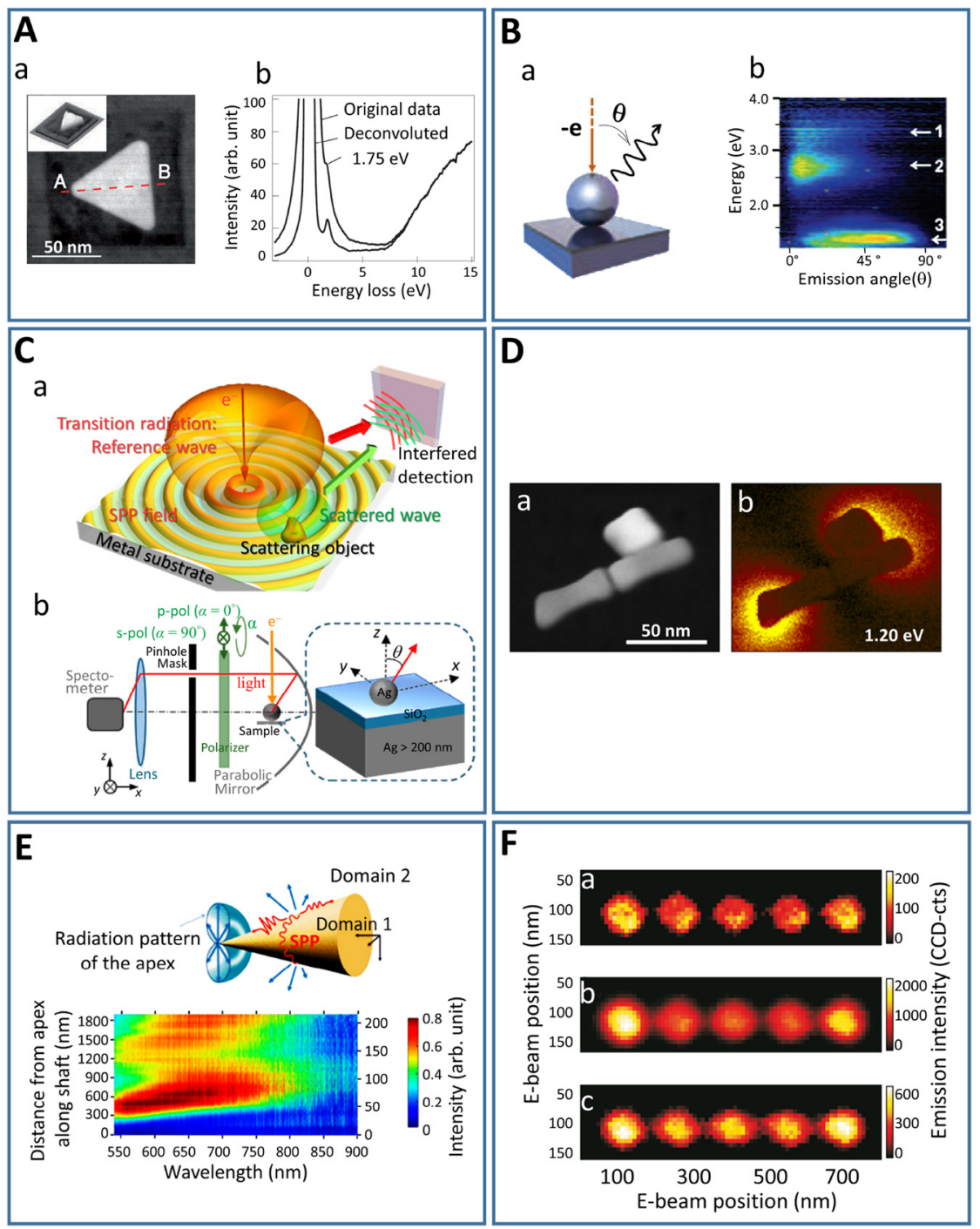

Figure 5: (A) (a) High-angle annular darkfield scanning transmission electron microscopy image of the Ag nanoprism with $78 \mathrm{~nm}$ long sides. (b) EEL spectra acquired at corner $\mathbf{A}$ before (raw data) and after deconvolution [76]. (B) (a) Schematics for excitation of gap mode by an electron beam. (b) Angular dependence of the emission from dipole Mie (2) and gap (3) plasmons in supported spheres [79]. (C) (a) Illustration of the method for the extraction of the SPP scattering phase through CL. Transition radiation (TR) from the metallic substrate is used as a reference wave, interfering in the far-field (at the detector) with out-coupled light resulting from the SPP interaction with the sampled object. (b) Illustration of the measurement setup, the sample, and the coordinate system [80]. (D) (a) Annular dark-field STEM image of a cluster of three gold particles consisting of two nanorods aligned end to end and an adjacent cube-shaped particle. (b) Map of the plasmon intensity at $1.20 \mathrm{eV}$ $(\lambda=1033 \mathrm{~nm})$, showing the resonance mode of the whole cluster [85]. (E) (Top) Schematic illustration of surface plasmon radiative decay in gold tapers. Red curves indicate the propagation of SPPs in forward, backward, and azimuthal directions. Blue lines depict the light emission due to the radiative decay of SPPs. (Bottom) Experimental CL spectra of a taper with a $13^{\circ}$ opening angle against the distance from the apex along its shaft (left) and the local radius (right) [91] (F) Spatially resolved excitation maps of the nanoantenna, showing $\mathrm{CL}$ intensity as a func-

tion of e-beam position for (a) $500 \mathrm{~nm}$, (b) $600 \mathrm{~nm}$, and (c) $750 \mathrm{~nm}$ detection wavelength integrated over a 10-nm bandwidth. The integration time per pixel is $0.1 \mathrm{~s}$ [45]. SPPs, surface plasmon polaritons; CL, cathodoluminescence; TR, transition radiation.

with opening angles of $13^{\circ}$ and $47^{\circ}$ have been investigated [91]. The three-dimensional gold tapers are investigated for fundamental mode order $m=0$ and higher-order modes | $m \mid>0$. CL was introduced to investigate plasmon-induced radiation of the gold tapers (Figure 5E). Interestingly, there was a weaker radiation from the apex of the $13^{\circ}$ taper compared to the $47^{\circ}$ taper. Simulations for the gold taper show that for small openings, plasmon modes created at the apex are efficiently guided along the tapered shaft with less radiation damping. In contrast, tapers with large openings experience larger radiation damping.

Plasmons supported by metals suffer from large attenuations due to dissipation and therefore cannot propagate to long ranges, in contrast with guided waves in a dielectric material. Although for devices with nanoscale dimensions, its relative small propagation length can be harnessed for technological applications. SPs play an important role in surface-enhanced Raman spectroscopy with potential applications in medical diagnostics [92]. They also play a role in explaining the anomalies of diffraction from metal gratings (wood's anomaly) [93]. This has led to the emergence of spoof plasmons [94, 95]. Metal particle assemblies functionalized with biomolecules can be used as a molecular scale ruler. The length scale measurable with metal particle assemblies is larger than the length scale measured using the fluorescence resonance energy transfer metrology method routinely used in biology [96]. Plasmons also find applications in the design of antennas $[97,98]$. The emission properties of a Yagi-Uda antenna composed of a linear array of $\mathrm{Au}$ nanoparticles have been studied experimentally using high-energy electron beams as a broadband point dipole source of 
visible radiation [45]. The experimental work showed that the directional emission of light depends strongly on the position where the antenna is excited (Figure 5F). This effect, together with the tunability of the resonances in nanoantenna versus their shapes and compositions, can be used for sensing chemical components and bio-molecules [99].

\subsection{Coherent bremsstrahlung radiation source}

A number of different scattering events such as Thompson scattering, bremsstrahlung scattering, Mott scattering, etc. occur when a moving electron interacts with materials. For electrons passing by the nucleus of the atoms, a radiation known as BR is produced. This is due to loss in the kinetic energy of the electron [100]. From Maxwell's equations, it is evident that an accelerating charged particle produces electromagnetic radiations. Thus, as the electron interacts with the nucleus, depending on its energy, electromagnetic radiation is produced usually in the X-ray region of the electromagnetic spectrum. Mathematical relations for the energy loss have both been derived classically and quantum mechanically [101-103]. An estimate for the average differential cross section per photon energy $\left(E_{\mathrm{p}}\right)$ can be calculated as follows [104, 105]:

$$
-\left(\frac{d \sigma}{d E_{\mathrm{p}}}\right) \simeq \frac{4 Z^{2} \alpha r_{e}^{2}}{E_{\mathrm{p}}} \ln \left(\frac{183}{Z^{\frac{1}{3}}}\right)
$$

where $r_{\mathrm{e}}=2.82 \times 10^{-15} \mathrm{~m}$ is the classical radius for the electron, $Z$ is the atomic number of the target material, $\alpha$ is the fine structure constant, $\hbar$ is the reduced Planck's constant, $c$ is the speed of light, and $m_{e}$ is the mass of electron. In this section, our focus will be on coherent bremsstrahlung (CB) radiation as a potential EDPHS. If the target material is crystalline, then the resulting spectrum will be noncontinuous, and the observed sharp peaks are referred to as $\mathrm{CB}$. The earliest discussions and theories on $\mathrm{CB}$ are discussed in the studies by Palazzi [106], Timm [107], and Ter-Mikaelian [108]. The interference phenomenon governing CB has been explained elsewhere [109, 110]. The first quantitative calculation of $\mathrm{CB}$ spectrum and polarization was given by Saenz and Überall [111] in a form that is close to experimental results [112, 113]. Several experimental studies of CB have been carried out with relative accuracies to the first Born approximation [114].

Akhiezer et al. [116] emphasized that for first Born approximation theory to be applicable for CB calculation, the condition that $\phi \gg\left(Z e^{2} / E d\right)^{\frac{1}{2}}=\phi_{c}$ must be satisfied, where $\phi$ is the angle between the incident electron and a major crystal axis, $\phi_{c}$ is the Linhard critical angle, $E$ is the electron energy, and $\alpha$ is the lattice plane spacing. Although, at much lower energies, the condition $\phi \gg \phi_{c}$ need not be satisfied for application of the first Born approximation theory. The authors described their experimental work with high-energy electrons of $7-10 \mathrm{MeV}$, using first Born approximation at an incident angle, $\phi=0$, along [ $\left[\begin{array}{lll}1 & 0 & 0\end{array}\right]$ axis of $\mathrm{Si}$ [115]. The condition $\phi \ll \phi_{c}$ is very important for describing the phenomenon of channeling radiation [116] at electron energies high enough so that the classical consideration is relevant. Channeling is the phenomenon whereby a beam of charged electron incident on a major critical axis, a set of crystal planes or between two of such planes at an initial small-angle $\phi$, will propagate along the axis or planes guided by the relevant screened Coulomb force exerted on it by the atoms of the axis or planes, thus achieving large depth penetration [117]. By applying classical mechanics in describing the channeling effect, Linhard [118] showed that the particles are trapped into a channeling state $\phi \leq \phi_{c}$, where $\phi_{c}$ is the Linhard critical angle. A particle in such a channeling state can be described quantum mechanically by a wave function, which can be thought of as being transversely bond with a nonzero probability of emitting spontaneous radiation. Beyond the first Born approximation, a more accurate calculation of the radiation emitted by the electron transversing a crystal can be evaluated from the first-order matrix for radiative transition [119]. This can be referred to as a second-order Born approximation.

$$
J_{\lambda}=\int\left\{\phi^{\prime{ }^{*}} \mathbf{p}^{\prime}(\mathbf{r})\left(\mathbf{u} \cdot \boldsymbol{\varepsilon}_{\lambda} e^{-i \mathbf{k} \cdot \mathbf{r}}\right) \phi_{\mathbf{p}}(\mathbf{r})\right\} d^{3} r
$$

where $\mathbf{p}$ and $\mathbf{p}^{\prime}$ denote the momentum of the initial and final electron, respectively, and $\mathbf{k}$ that of the photon, whose polarization vector is denoted by $\boldsymbol{\varepsilon}_{\lambda}, \mathbf{u}$ is the vector whose components are the usual 4 by 4 dirac matrices, the electron wave functions $\phi_{\mathbf{p}^{\prime}}^{\prime}(\mathbf{r}), \phi_{\mathbf{p}}(\mathbf{r})$ are taken as four component spinors which are of the bloch form, and the integration is taken over the volume of the crystals. Thus

$$
\phi_{\mathbf{p}}(\mathbf{r})=e^{i \mathbf{p} \cdot \mathbf{r}} U_{\mathbf{p}}(\mathbf{r}), U_{\mathbf{p}}(\mathbf{r}+L)=U_{\mathbf{p}}(\mathbf{r})
$$

where $L$ is the vector for the direct lattice and similarly for $\phi_{\mathbf{p}^{\prime}}^{\prime}(\mathbf{r})$. Saenz and Überall [112] put forth that CB radiation and the phenomenon of channeling radiation are two aspects of the same physical process. These two types of radiation can be described by the Bloch wave function in a unified framework. The Bloch wave function given by Eq. (3) is considered to be of high energy state. They can be expanded into a plane wave plus correction terms representing distortion of the plane wave by the periodic crystal 
potential. The first Born approximation to $J_{\lambda}$ is due to the correction term proportional to the electric charge [120]. However, applying the perturbation expansion for calculating CB emission becomes ineffective since the radiation involves a transition between transversely bond states that can be approximated by Bloch plane waves. Hence, CB and channeling radiation are two different aspects of the same phenomenon, depending on whether the Bloch wave functions of the electron are analogous to channeling states or close to free plane wave states. The radiative transition in a crystal may be classified as free-free transition (CB), band-to-band transition (channeling radiation), and also free-bond transition. These radiation types can be experimentally differentiated via kinematics, through the dependencies of their transition energies on the incidence angle between the electron on the crystals [121]. Finally, we highlight that despite the potential of CB for designing appropriate X-ray EDPHSs, still, this is a path to be trailed.

\subsection{Smith-Purcell radiation}

The Smith-Purcell effect involves the generation of a continuum of electromagnetic radiation with varying direction as free electrons propagate along the surface of a metallic grating. This phenomenon was first observed by Smith and Purcell [122] in 1953. They send an electron beam with the kinetic energy of $300 \mathrm{keV}$ along the surface of a grating and observed the emission of visible radiation. Using Huygens principle, they found a mathematical relationship between the velocity of the electron and wavelength of the emitted light as follows:

$$
\lambda=\frac{L}{|m|}\left(\frac{1}{\beta}-\cos \theta\right)
$$

where $L$ denotes the grating period and $m$ is the order of diffraction. $\lambda$ denotes the wavelength of the radiation produced at angle $\theta, \beta=\frac{v}{c}$, where $v$ is the velocity of the electron and $c$ is the speed of light. Thus, by tuning the velocity of the electron beam sources, such as thermionic and field emission sources in scanning electron microscopes, the wavelength of the emitted light can be modified. The concept of SP radiation has been developed more than a century ago. There have been insightful theoretical and experimental approaches discussing SP radiation. The geometry of the metallic grating that leads to the production of SP radiation can take different forms. This influences whether an analytical formula will be obtainable or not for a particular SP process. Analytical expression for the radiation emission probability and electron energy-loss spectra, for finite and infinite strings of $\mathrm{Al}$ and $\mathrm{Si}$ nanospheres, have been discussed [123]. The author obtained the analytical solution for the previously defined quantities by solving the Maxwell equation exactly, using the multipole expansion method. Technical details regarding SP radiation will be discussed in detail in Design of possible EDPHSs.

\section{Design of possible EDPHSs}

\subsection{Metamaterial-based sources}

Metamaterials have been extensively studied in the past decade. Metamaterials are artificially engineered structures with tailored electromagnetic properties [52, 124]. By using localized plasmonic modes and probing them with electromagnetic radiation, a host of unique phenomena have been uncovered by various researchers [125-127]. As an application, chiral materials can rotate the polarization of light with tailored properties [128]. It has been experimentally demonstrated that localized injection of electrons into a large ensemble of planar plasmonic metamolecules drives directed light emission [129]. The emission emanates from the collective coupled oscillation of the large metamolecule array (Figure 6A). The metamolecule design comprises square arrays of asymmetric split rings (see inset Figure 6C). The light emission is analogous to laser light in the sense that it sustains coherent light of tailored properties. Figure $6 \mathrm{~B}$ shows the emission spectra for different sizes of metamolecules while Figure 6C shows the absorption spectra for different cell sizes. The temporal coherence of the emitted radiation increases with increasing size, and the resonant metamaterial emission is directional. Also, Adamo et al. $[130,131]$ showed that electron beams interacting with a metamaterial planar structure leads to the emission of photons that are coherent with the near field of the electron.

The EDPHS, which finds application in electron spectroscopy, can be designed with metamaterials. The flexibility of manipulating metamaterials for desired properties makes them highly advantageous for the design of coherent EDPHSs. The electromagnetic properties of metamaterials can be altered by manipulating the shape of the individual resonators or by manipulating near-field interaction between them. Zheludev and Kivshar showed that by continuous adjustment of the lattice structure, an efficient approach for tuning the transmission characteristics of the metamaterial was found [132]. Reconfigurable metamaterials via reconfiguring the metamolecules have been achieved using microelectromechanical systems [133]. This is done with the outlook that controlling the resonant properties of the individual elements could be used to make tunable refractive index material arrays. In the 

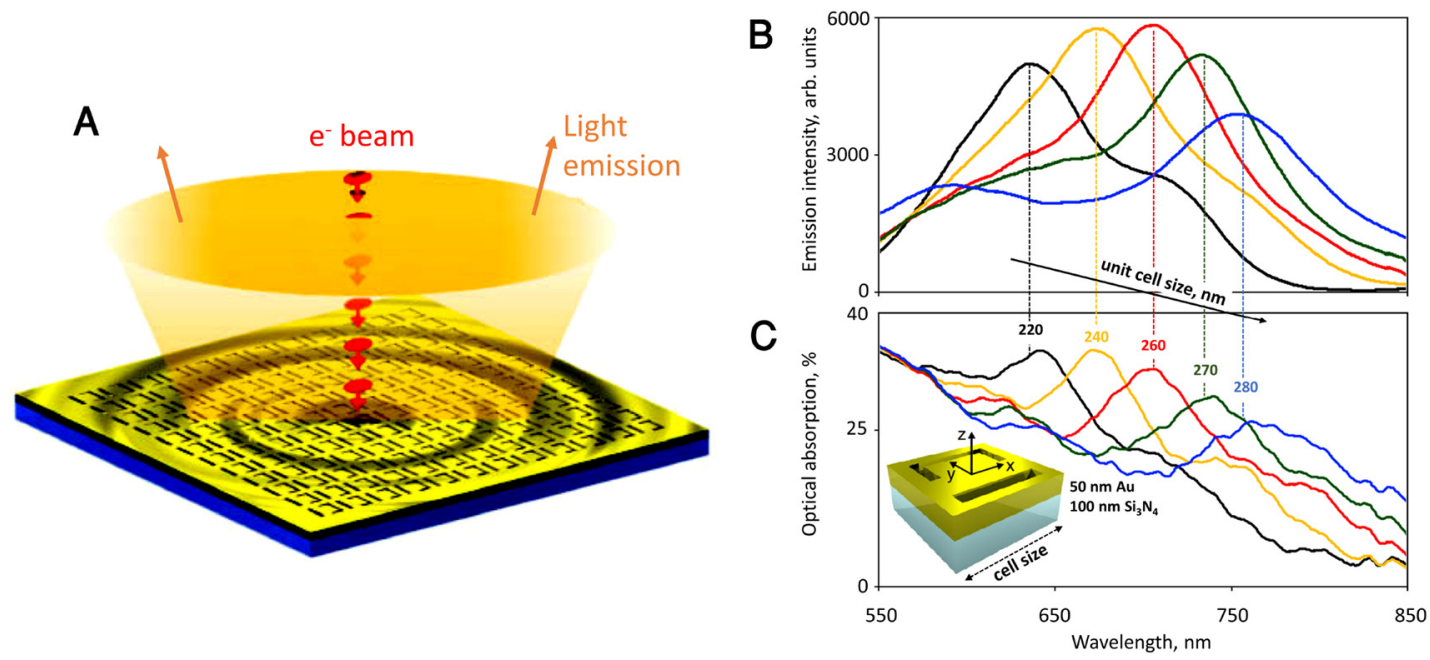

Figure 6: (A) Highly localized electron beam excitation at the center of a metamaterial array leads, via strong coupling among metamolecules, to the collective oscillation of many cells and thereby the emission of a free-space light beam. (B) Electron beam-induced metamaterial light emission spectra for arrays with different metamolecules size (as labeled). (C) Corresponding optical absorption spectra. The inset to $C$ shows a schematic of the asymmetric split-ring metamolecules [130].

terahertz frequency range, reconfigurable metamaterials have been designed by fabricating planar arrays of splitring resonators on bimetallic cantilevers [134]. Nanostructure material hybridized with nonlinear and switchable dielectric or semiconductor layers and controlled with ultrafast optical pulses provides faster switching compared to micromechanical repositioning [135]. Reconfigurable metamaterial can be used for designing EDPHSs with controllable and on-demand light properties.

Hyperbolic metamaterials are an interesting class of metamaterials with tailored properties. Due to the relative ease of fabrication, wavelength tunability, broadband nanoresponse, hyperbolic metamaterials have various applications for nanophotonic systems [136, 137]. Hyperbolic materials can occur naturally or be engineered artificially. A detailed review of natural and man-made hyperbolic media is discussed elsewhere [138]. Moritz et al. expounded on tetradymites, a natural hyperbolic material for the near field to the visible spectrum of the light $[51,139]$. They extensively discussed two classes of tetradymites compounds, $\mathrm{Bi}_{2} \mathrm{Se}_{3}$ and $\mathrm{Bi}_{2} \mathrm{Te}_{3}$. These compounds are layered van der Waals materials which account for its strong anisotropic behaviors. Although not yet studied, tetradymites show very interesting properties desired for the fabrication of EDPHSs.

\subsection{Smith-Purcell sources}

SP radiation sources can be coherent or incoherent. This depends on several factors such as the shape of the electron wave function and nature of the material involved [52]. There have been reports on enhanced coherent SP radiation at terahertz frequency from a train of picosecond bunches of $15 \mathrm{MeV}$ passing above a grating [22]. The authors showed a trivial method for generating powerful terahertz radiation by observing SP radiation enhanced by the number of electrons in the bunch, the number of bunches in a train, and the number of grating periods (Figure 7A). According to Andrews et al. [140], coherent SP radiation dominates if the electrons are in bunches. The radiation intensity varies as the square of the number of electrons present in the bunch. If the bunches occur periodically [141, 142], then the phenomena are called superradiance [140]. The radiated energy density from a single electron moving parallel to a grating per unit frequency for the $n$th order SP radiation is given as follows [143]:

$$
\frac{d I_{n}}{d \omega}=\frac{e^{2} N_{e} l}{8 \pi^{2} \varepsilon_{o} c^{2}} \omega \sin ^{2} \theta \int\left|R_{n}\right|^{2} e^{-\frac{x_{o}}{\lambda_{e}}} \sin ^{2}(\phi) d \phi
$$

where $x_{o}$ is the height of the electron above the grating, $\phi$ is the azimuthal angle, $l$ is the grating lenght, $\varepsilon$ is free space permitivity, $c$ is the speed of light, $\left|R_{n}\right|^{2}$ is the grating efficiency factor, and $\lambda_{e}$ is the evanescent wavelength which is defined as follows:

$$
\lambda_{e}=\lambda \frac{\beta y}{4 \pi \sqrt{1+\beta^{2}\left(1-\beta^{2}\right) \sin ^{2} \theta \sin \phi}}
$$

However, for $N_{e}$ electrons in a bunch, the total radiated intensity takes the following form: 


$$
\left(\frac{d I_{n}}{d \omega}\right)_{N_{e}}=\left(\frac{d I_{n}}{d \omega}\right)\left(N_{e} S_{i n c}+N_{e}^{2} S_{c o h}(\omega)\right)
$$

where $S_{\text {inc }}$ and $S_{\text {coh }}(\omega)$ denote the incoherent and coherent radiation contribution, respectively. Superradiance takes place at the bunch frequencies or its integer multiple. It is important to add that the emission occurs only at the corresponding SP angles. Several simulations and theoretical works have been carried out to investigate this phenomenon [144-146]. The particle-in-cell code has been applied in the study of SP radiation [145]. The model supposes a rectangular grating with a period of $173 \mu \mathrm{m}$ to be driven by a
A

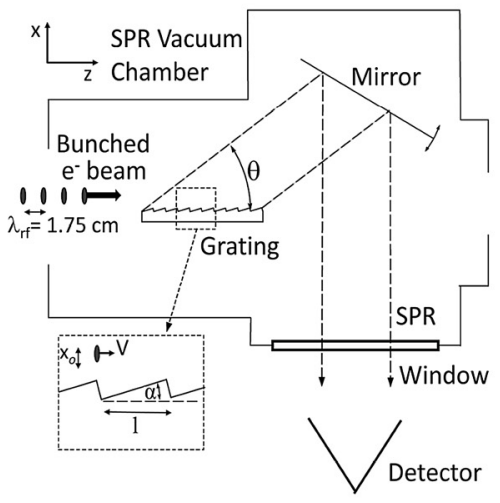

C

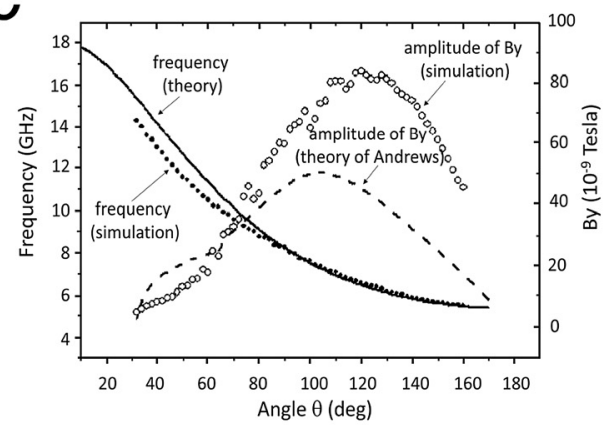

$E$

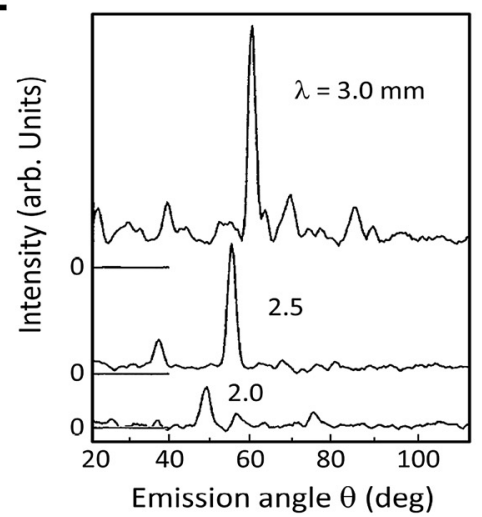

B

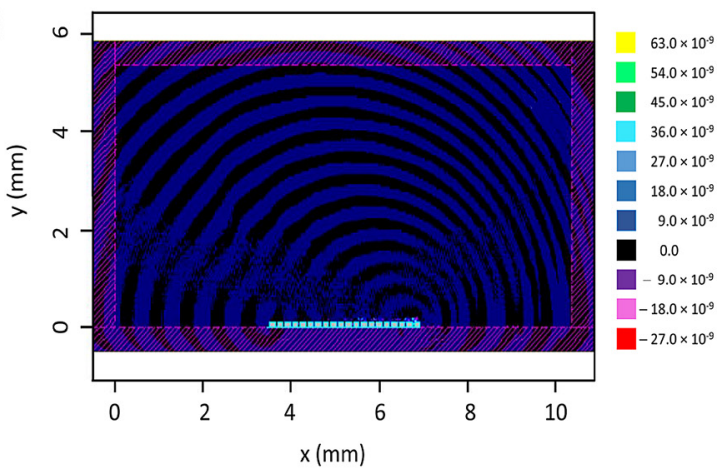

D

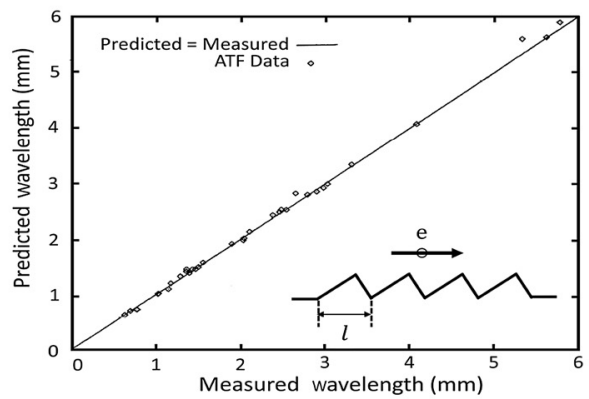

$\mathbf{F}$

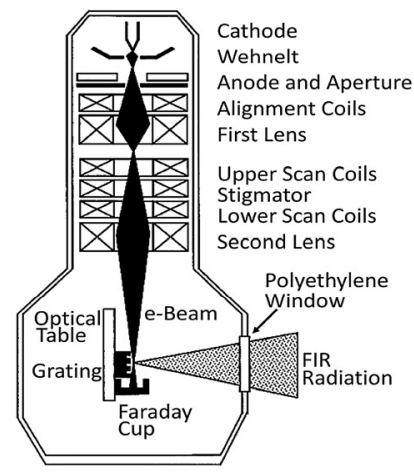

Figure 7: (A) SP radiation experimental setup. The train of bunched electrons is traveling along the $z$ direction above a metallic echelle grating. The diffracted radiation is measured by a detector located outside of the vacuum chamber [22]. (B) Crescent-shaped wavefronts of coherent SP radiation observed via the contour plot of the magnetic field $B_{z}$ at $t=65.6 \mathrm{ps}$ [146]. (C) The dependence of SP frequency and amplitude of the magnetic field $B_{y}$ on the emission angles, observed at distances $36.35 \mathrm{~cm}$ from the center of the grating [148]. (D) Wavelength measurements for aluminum with $30^{\circ}$ blaze grating which has periods of $1,2,4 \mathrm{~mm}$ and $20^{\circ}$ blaze grating with periods of $2,4 \mathrm{~mm}$ for angles between $18^{\circ}-40^{\circ}$. The plot shows good agreement between predicted and experimental wavelength [150]. (E) Dependence of the coherent SP radiation intensity on the emission angle. The intensity was measured with the $42 \mathrm{MeV}$ short-bunched electron beam passing over a $6 \mathrm{~mm}$ period grating at the wavelengths 2.0, 2.5, and $3.0 \mathrm{~mm}$ [156]. (F) Schematic of the modified scanning electron microscope for generating coherent SP [157]. SP, surface plasmon. 
single electron bunch, a train of periodic bunches, and a continuous beam, respectively (Figure 7B). In another work, the authors carried out a three-dimensional simulation to investigate SP radiation using the Magic code [147] (Figure 7C). They compared their obtained result with theory and found reasonable agreement between them. SP radiation has also been studied experimentally [21, 148]. The first experimental evidence for the generation of forward-directed SP emission was discussed in the study by Woods et al. [149]. An aluminum grating of $1.69 \mathrm{~cm}$ wide and $17 \mathrm{~cm}$ long with a period of $1.0 \mathrm{~cm}$ and a blazed angle of $5^{\circ}$ was used to investigate the forward SP emission (Figure 7D).

It is generally accepted that blazed, compound, and complex gratings can be harnessed for the design of spectral and spatial distribution of transmitted, reflected, and guided light modes with improved desired properties such as enhancement or improved coherence of emitted radiation [150]. These kinds of grating can be used for beam shaping [151, 152], spectroscopy [153], and spectral filtering [154]. Coherent SP radiation, generated by the passage of relativistic short-bunched electrons across the surface of a metallic grating, has been observed in the wavelength region from 0.5 to $4.0 \mathrm{~mm}$ [155]. By changing the angle between the relativistic electron and the grating, the angular distribution of the radiation was measured. Figure $7 \mathrm{E}$ shows the angular distribution obtained at wavelength 2.0, 2.5 , and $3.0 \mathrm{~mm}$, respectively. with a grating of $6 \mathrm{~mm}$ period. At each wavelength, a principal peak and several weak peaks are seen which is in agreement with theory. Urata et al. [156] present grating-based coherent tunable sources that operate in the far-infrared region of the spectrum. By incorporating a low energy spread, low emittance electron beam, and a diffraction grating to a scanning electron microscope, they pushed the operating regime of the scanning electron microscope toward the far-infrared $30-1000 \mu \mathrm{m}$ region of the spectrum (Figure $7 \mathrm{~F}$ ).

\subsection{Holographic sources}

Holography is a unique method for manipulating the wavefront of light. It was first invented in 1948 by Gabor [157] whose motivation was to use the holographic technique to improve the resolution of electron microscopes. The technique involves recording and reconstruction of the full-wave information of a target object including image amplitude as well as its phase by a detector. Interference patterns between the reference wave and scattered wave create the hologram at the detector. This is the principle behind inline holography, and electron and optical spectroscopy analysis makes use of this principle. The advent of the lasers paved the way for holographic principles to be applied to free-space optical beams [158]. Holographic techniques have evolved in the field of optics as a unique way of manipulating image information. Brown and Lohman [159] developed the computer-generated holograms. They used numerical computation to calculate the phase information of the wave at the hologram interface. This enabled creation of virtual objects [160], and the concept was extended by Cowan [161] from free surface waves to free-space waves. Recently, methods for achieving optical holography have been influenced by novel nanofabrication techniques [162]. Subwavelength tailored devices such as gratings, photonic crystals, metamaterials, and metasurfaces are opening new horizons for holographic and optical devices such as beam splitter and image processing metasurfaces [163]. The two-dimensional form of metamaterials, the metasurface, provides exceptional control over the propagation of light [164-166].

Metasurface holography offers unique methods for controlling SPP waves and free waves $[167,168]$. They can be used in the manipulation of EDPHSs to achieve coherent and focused TR. Li et al. [169] introduced a universal approach for the generation of light with defined wavelength, direction, divergence, and topological charge through point excitation of the holographic plasmonic metasurface. They were able to show that the interaction between the metasurface and free electrons generated highly directional visible to near-infrared beams in described azimuthal and polar directions (Figure 8A). The holographic design approach was used to generate complex wavefronts (such as vortex beam generation). Figure 8B shows the central part portions of the binary holographic mask for the generation of vortex beams with topological charge $l=3,6$, 9 while Figure $8 \mathrm{C}$ shows the farfield distribution of light emitted from the holographic nanostructure of similar angular momentum. The holographic techniques can serve as a coherent source of radiation.

\subsection{Photon sieve sources}

Photon sieves are diffractive lens used for focusing planar X-rays [170]. Photon sieves usually consist of pinholes distributed in the Fresnel zones. The pinholes are of diameter comparable to the wavelength of light. Kipp et al. [171]s proposed this concept in 2001. The photon sieve proposed by Kipp et al. [171] comprises 5646 randomly distributed holes and was very complicated. The design details regarding the sieve fabrication were not explicitly 
A

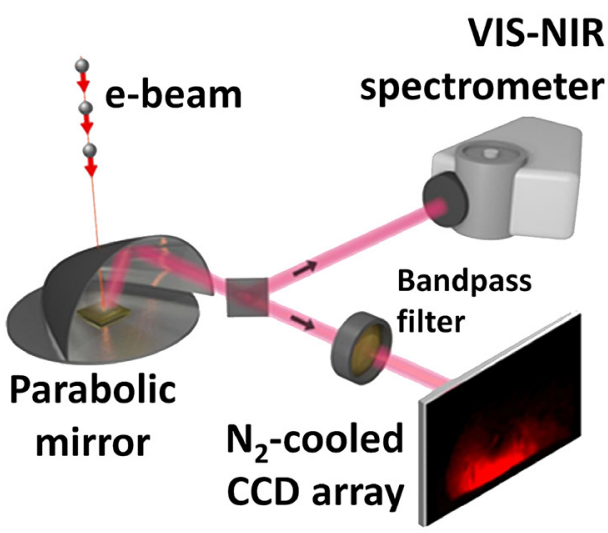

B

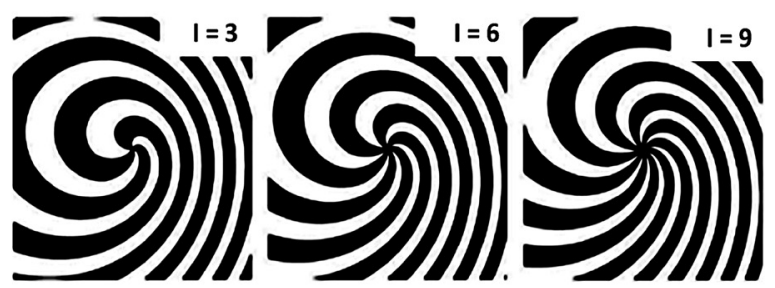

C

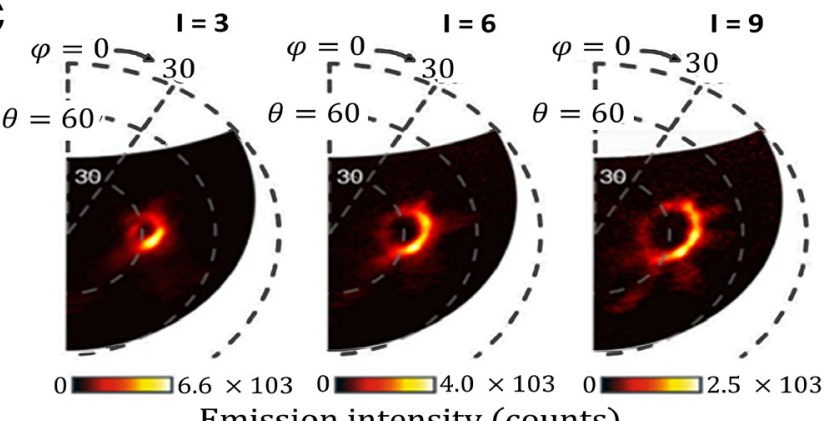

Emission intensity (counts)

Figure 8: (A) Schematic of the scanning electron microscope-based system for angle-resolved electron-induced light emission spectroscopy. (B) Central portions of binary holographic masks for electron beam-induced generation of optical vortex beams with topological charge at $l=3,6$, and 9. (C) Corresponding angle-resolved emission intensity distribution maps (electron beam current $=5.5-6.1 \mathrm{nA}$; integration time $=30 \mathrm{~s})[170]$.

captured in their paper. The distributed pinholes in photon sieve structures are designed in such a way that the emergent rays from the structure interferes constructively in the far-field. This implies that certain conditions need to be fulfilled; the optical path length of the photon from the source through the center of the pinholes to the focal point should be an integer value of the wavelength, $\lambda$. This condition is captured by the following equation [171]:

$$
\sqrt{r_{n}^{2}+p^{2}}+\sqrt{r_{n}^{2}+q^{2}}=p+q+n \lambda
$$

where $r_{n}$ is the distance between the center of the holes and optical axis, $p$ is the distance between the source and photon sieve, $q$ is the distance between the photon sieve and the focal point. Theories governing photon sieve focusing dynamics in the nonparaxial mode and far-field have been discussed elsewhere [172, 173].

Fresnel zone plates (FZPs) also have focusing capabilities. They have been used for focusing X-rays for a long time $[174,175]$. In image formation, FZPs suffer from blurred images due to the formation of ring-shape secondary maxima observed at the focal plane. This challenge can be circumvented by controlling the width of the outermost zone. This poses engineering challenges when approaching the high-resolution zone. On the other hand, photon sieves enable sharper images by suppressing these secondary maxima, circumventing processing limitation of the resolution of FZPs. The focusing properties of the photon sieve structure are superior compared to normal rectangular zone plates and show a pronounced decrease in peak width and side lobes (Figure 9C). However, the transmission capacity for photon sieve is lesser than that for FZPs. The transmission capacity for the amplitude zone plate is typically $50 \%$ while that of photon sieve is about $15-20 \%$ [172]. The transmission capacity for both the FZP and photon sieve depends on the size of the pinholes and the opening area of the zones. Several other photon sieve structures have been proposed to create structured light, such as chiral light [176-179]. One unique property of the EDPHS structure is that it should produce focused radiation that couples the target material to the far-field. Thus, designing the EDPHS structure based on the photon sieve concept satisfies these criteria. Besides, the structural complexity of metamaterial and holographic based EDPHS structure can be circumvented by using more efficient design principles such as photon sieve concepts. The succeeding section depicts how we took advantage of the photon sieve concept for EDPHS design.

\subsubsection{Focused radiation}

A different design principle for the distribution of pinholes is to be employed when it is desired to shape or focus the radiation from electron beams. An electron only emits light on interaction with the structure, according to the radiation mechanisms described in previous sections. For the design of EDPHSs, it is generally required that the generated 


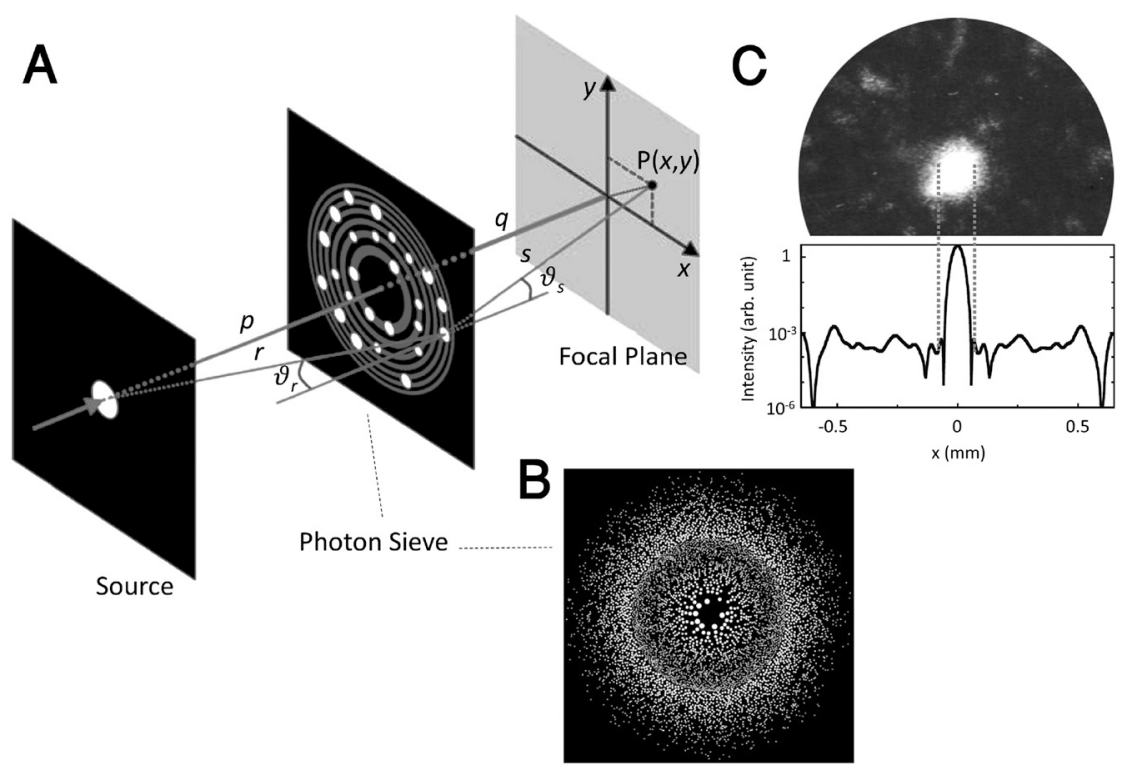

Figure 9: Photon sieve structure for focusing planar light radiation. (A) The design principle for achieving constructive radiation at the focal plane (B) A fabricated photon sieve structure comprising 5646 transmissive pinholes (C) The intensity at the focal plane [172]. radiation is focused over a broad spectral range; however, a diffractive design principle based on positioning pinholes on diffractive zone plates generally only focuses monochromatic light. Hence, a metallic thin film that generates SPPs on interaction with the electron has been introduced. The mechanism to focus the generated plasmon-induced radiation using structural topology over a broad spectral range is also discussed [66].

Electron-induced SPs do not contribute to the emitted radiation, unless they interact with engineered defects, such as a hexagonal lattice of nanopinholes. The emissions in general mimic the symmetry of the structure. The electron-induced radiation, generated by the electron beam interacting with the hemispherical curved geometry comprising a hexagonal lattice of holes, is focused at the center of the hemisphere (Figure 10A). This depicts a broadband characteristic; hence, a spherical geometry acts as a lens for electron-induced radiation by curving the wavefronts into a spherical wavefront. By projecting the position of pinholes from a spherical coordinate system to a plane in the Cartesian coordinate system, a planar EDPHS is thus achieved (Figure 10B). The fabricated photon sieve structure (EDPHS) comprises pinholes with diameters of $100 \mathrm{~nm}$, which is well beyond the diffraction limit of light, in contrast to photon sieve structures that focus planar waves. The lens might be described in an effective medium approximation, as an anisotropic planar film of graded refractive index, thus approaches the behavior of a curved film. The Raith ionline Plus system, a dedicated highresolution ion beam structuring tool, was used for the design. Using a focused beam of doubly charged gold ions
$\left(\mathrm{Au}^{++}\right)$at an acceleration voltage of $35 \mathrm{kV}$ with a beamlimiting aperture size of $7 \mu \mathrm{m}$, holes in nanometer order were milled through the structure (see Figure 10C for the scanning electron microscopy image of the source). The fabricated structures were characterized both numerically and experimentally, using CL angle-resolved mapping techniques. The simulation results depict the ability of the source to focus electron-induced radiation over a broad spectral range at a distance of $5.6 \mathrm{~mm}$ from the sources (Figure 10D). The angle-resolved CL map of the structures comprise concentric rings, revealing divergence of optical rays from the focal plane to the far-field [66].

\subsubsection{Structured light}

The hole distribution in photon sieve structures can be designed in a way to facilitate arbitrary structured light on interaction with electron beams (Figure 11). Such kinds of structures will be discussed here. We realized these structures by milling nanopinholes into a bilayer $\mathrm{Au} / \mathrm{Si}_{3} \mathrm{~N}_{4}$ structure composed of $40 \mathrm{~nm}$ evaporated gold films and $20 \mathrm{~nm} \mathrm{Si}_{3} \mathrm{~N}_{4}$ transmission electron microscopy membranes. The distributions of pinholes, as well as their sizes compared to the wavelength of emitted photons, are important factors to consider for achieving structured light. These structures can generate radially polarized emitted light of various intensity profiles. It is also possible to generate vortex light with Laguerre-Gauss-like spatial profiles of various transverse electric and transverse magnetic polarization, as will be discussed in another publication. 


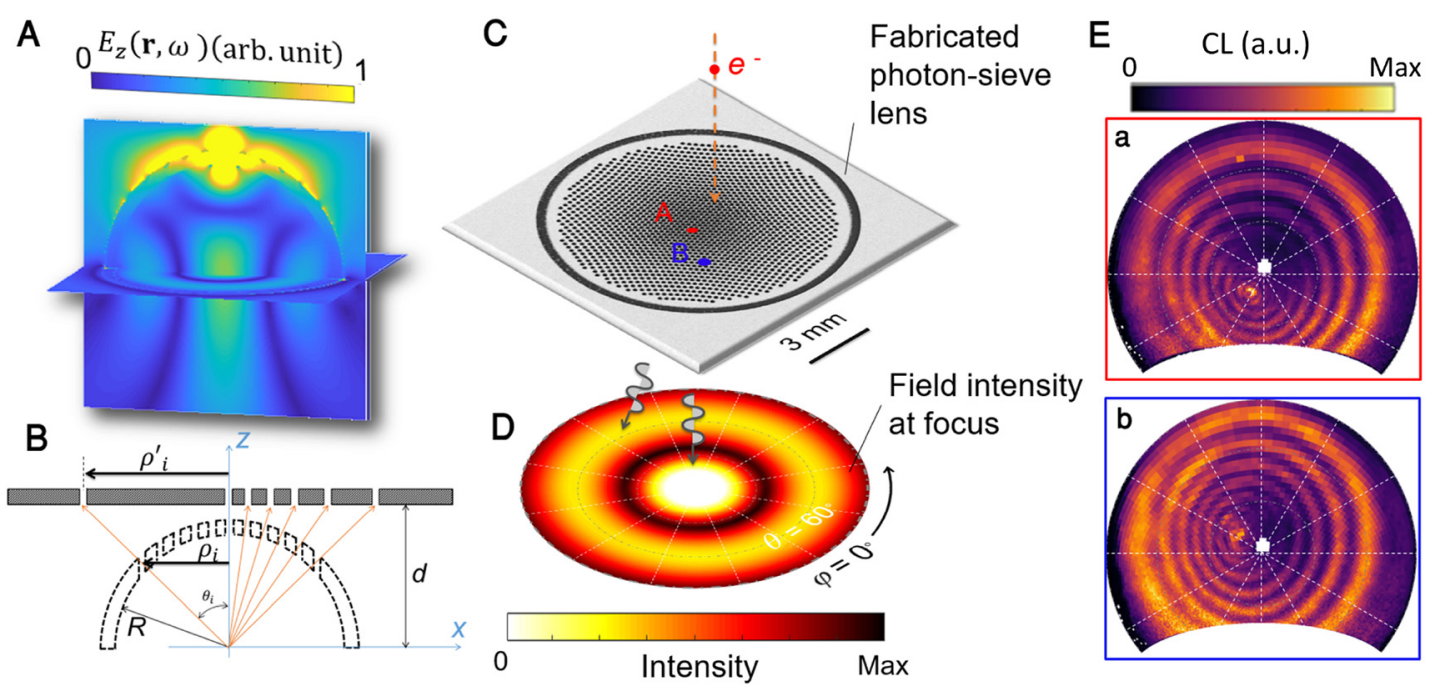

Figure 10: (A) Focused light from a hemispherical gold thin film comprising a hexagonal lattice of nanoholes interacting with an electron beam at the kinetic energy of $200 \mathrm{eV}$. (B) The pinholes are projected from spherical to Cartesian coordinate system to construct planar electrondriven photon sources with focused radiation. (C) Scanning electron microscope image of the fabricated photon sieve. (D) Calculated electric field intensity at the focal point situated at the distance of $5.6 \mathrm{~mm}$ from the structure. (E) Angle-resolved cathodoluminescence maps at different electron impact positions marked by $\mathrm{A}$ and $\mathrm{B}$ in the panel at $10 \mathrm{C}$, at the energy of $E=1.6 \mathrm{eV}[66]$.

The far-field radiation pattern of photon sieve structures can be characterized using an angle-resolved CL map. Delmic SPARC CL ${ }^{1}$ detector installed in a Zeiss SIGMA field emission scanning electron microscope ${ }^{2}$ was used for experimental analysis of these structures. Photon sieve with fourfold structural symmetry was created by projecting a square lattice of varying holes from the Cartesian coordinate system to a spherical coordinate system (Figure 11A). The obtained CL map comprises fourfold symmetry, with peak intensities forming into hyperbolic lines (Figure 11E and I). The CL maps at various wavelengths support the same symmetry profile, though with varying number of hyperbolic lines. Structures with sixfold symmetry obtained by mapping pinholes of a hexagonal lattice from the spherical coordinate system to a Cartesian coordinate system have this behavior (Figure 11B).

A method based on the interaction of radially polarized plasmons by the nanopinholes was employed to facilitate a faster designing procedure for arbitrary structured light. This method was adopted with the consideration that the ineraction results only in the modulations of the induced current density distribution on the surface of nanopinholes. The wave function associated with the radially polarized plasmons can be described by Bessel functions. The equivalence principle was used to obtain the electric and magnetic current density distribution on the surface of

$$
\begin{aligned}
& \frac{\left\{-i \sqrt{\varepsilon_{r}^{M} k_{o}^{2}-k_{\rho}^{2}} \sin \left(\sqrt{\varepsilon_{r}^{M} k_{o}^{2}-k_{\rho}^{2} d}\right)-\varepsilon_{r}^{M} \sqrt{k_{o}^{2}-k_{\rho}^{2}} \cos \left(\sqrt{\varepsilon_{r}^{M} k_{o}^{2}-k_{\rho}^{2} d}\right)\right\} \sqrt{\varepsilon_{r}^{M} k_{o}^{2}-k_{\rho}^{2}}}{\left\{\sqrt{\varepsilon_{r}^{M} k_{o}^{2}-k_{\rho}^{2}} \cos \left(\sqrt{\varepsilon_{r}^{M} k_{o}^{2}-k_{\rho}^{2} d}\right)+i \varepsilon_{r}^{M} \sqrt{k_{o}^{2}-k_{\rho}^{2}} \sin \left(\sqrt{\varepsilon_{r}^{M} k_{o}^{2}-k_{\rho}^{2} d}\right)\right\} \varepsilon_{r}^{M}} \\
& =\frac{\left\{-i \sqrt{\varepsilon_{r}^{D} k_{o}^{2}-k_{\rho}^{2}} \sin \left(\sqrt{\varepsilon_{r}^{D} k_{o}^{2}-k_{\rho}^{2} h}\right)-\varepsilon_{r}^{D} \sqrt{k_{o}^{2}-k_{\rho}^{2}} \cos \left(\sqrt{\varepsilon_{r}^{D} k_{o}^{2}-k_{\rho}^{2} h}\right)\right\} \sqrt{\varepsilon_{r}^{D} k_{o}^{2}-k_{\rho}^{2}}}{\left\{\sqrt{\varepsilon_{r}^{D} k_{o}^{2}-k_{\rho}^{2}} \cos \left(\sqrt{\varepsilon_{r}^{D} k_{o}^{2}-k_{\rho}^{2} d}\right)+i \varepsilon_{r}^{D} \sqrt{k_{o}^{2}-k_{\rho}^{2}} \sin \left(\sqrt{\varepsilon_{r}^{D} k_{o}^{2}-k_{\rho}^{2} h}\right)\right\} \varepsilon_{r}^{D}}
\end{aligned}
$$

1 https://www.delmic.com/en/products/cl-solutions/sparc-spectral. $2 \mathrm{https} / / /$ www.zeiss.com/microscopy/int/products/scanningelectron-microscopes/geminisem/fe-sems-with-gemini-optics.html. the structure at ( $z=d$ surface). The electric and magnetic current densities associated with the radially polarized surface plasmons are obtained as follows: 


$$
\begin{gathered}
\vec{J}(\rho, \omega)=\widehat{z} \times \vec{H}_{\phi}(z=d, w)=k_{\rho} H_{o}^{(2)^{\prime}}\left(k_{\rho}\right) \hat{\rho} \\
\vec{M}(\rho, \omega)=-\widehat{z} \times \vec{E}_{\rho}(z=d, w)=\frac{\sqrt{k_{o}^{2}-k_{\rho}^{2}}}{\omega \varepsilon_{o}} k_{\rho} H_{o}^{(2)^{\prime}}\left(k_{\rho}\right) \widehat{\phi}
\end{gathered}
$$

where $\vec{H}_{\phi}$ and $\vec{E}_{\rho}$ are the $\phi$ and $\rho$ components of the magnetic and electric fields. $\omega$ is the angular frequency, $\varepsilon_{o}$ is the free space permittivity, and $k_{\rho}$ is the phase constant to be obtained using the boundary conditions. Some straight forward algebra yields the following characteristics equation for calculating $k_{p}$ :

where $\varepsilon_{r}^{D}$ and $\varepsilon_{r}^{M}$ are the dispersive permittivities of $A u$ and $\mathrm{Si}_{3} \mathrm{~N}_{4}$, respectively, $D$ is the thickness of the gold layer, and $h$ is the thickness of $\mathrm{Si}_{3} \mathrm{~N}_{4}$ membrane, and $k_{o}$ is the freespace wavenumber of the light. Far-field radiation is assumed to be generated as a result of the interaction of surface plasmons with nanopinholes. Therefore, the photon sieve structures act as an intensity mask for the radiation in such a way that the current distributions only at the position of nanopinholes are assumed nonzero. The intensity profile of the radiation at the far-field was obtained from the free-space Green function. The vector potentials were obtained as follows:

$$
\vec{A}(x, y, z)=-\iint d k_{x} d k_{y} \overrightarrow{\tilde{J}}\left(k_{x}, k_{y} ; \omega\right) e^{-i k_{x} x} e^{-i k_{y} y} e^{-i \sqrt{k_{o}^{2}-k_{x}^{2}-k_{y}^{2} z}}
$$

and

$$
\vec{F}(x, y, z)=-\iint d k_{x} d k_{y} \overrightarrow{\tilde{M}}\left(k_{x}, k_{y} ; \omega\right) e^{-i k_{x} x} e^{-i k_{y} y} e^{-i \sqrt{k_{o}^{2}-k_{x}^{2}-k_{y}^{2} z}}
$$

for electric and magnetic vector potentials, respectively. Electric field was obtained as follows:

$$
\vec{E}(\vec{r})=-\vec{\nabla} \times \vec{F}(\vec{r})+\left(i \omega \varepsilon_{o}\right)^{-1} \times(\vec{\nabla} \times \vec{\nabla} \times \vec{A}(\vec{r})-\vec{J}(\vec{r}))
$$

The CL emission was calculated as the intensity of the transversal electric field at the far-field, with the results shown in Figure 12. A direct comparison between our simplified theory and the experimental results shown in Figure 11 demonstrates that our theory works well in highlighting significant features of the angle-resolved maps and might be used for designing photon sieve structures to facilitate on-demand structured light.

In addition to structural symmetry and composition of nanopinholes, an additional degree of freedom for
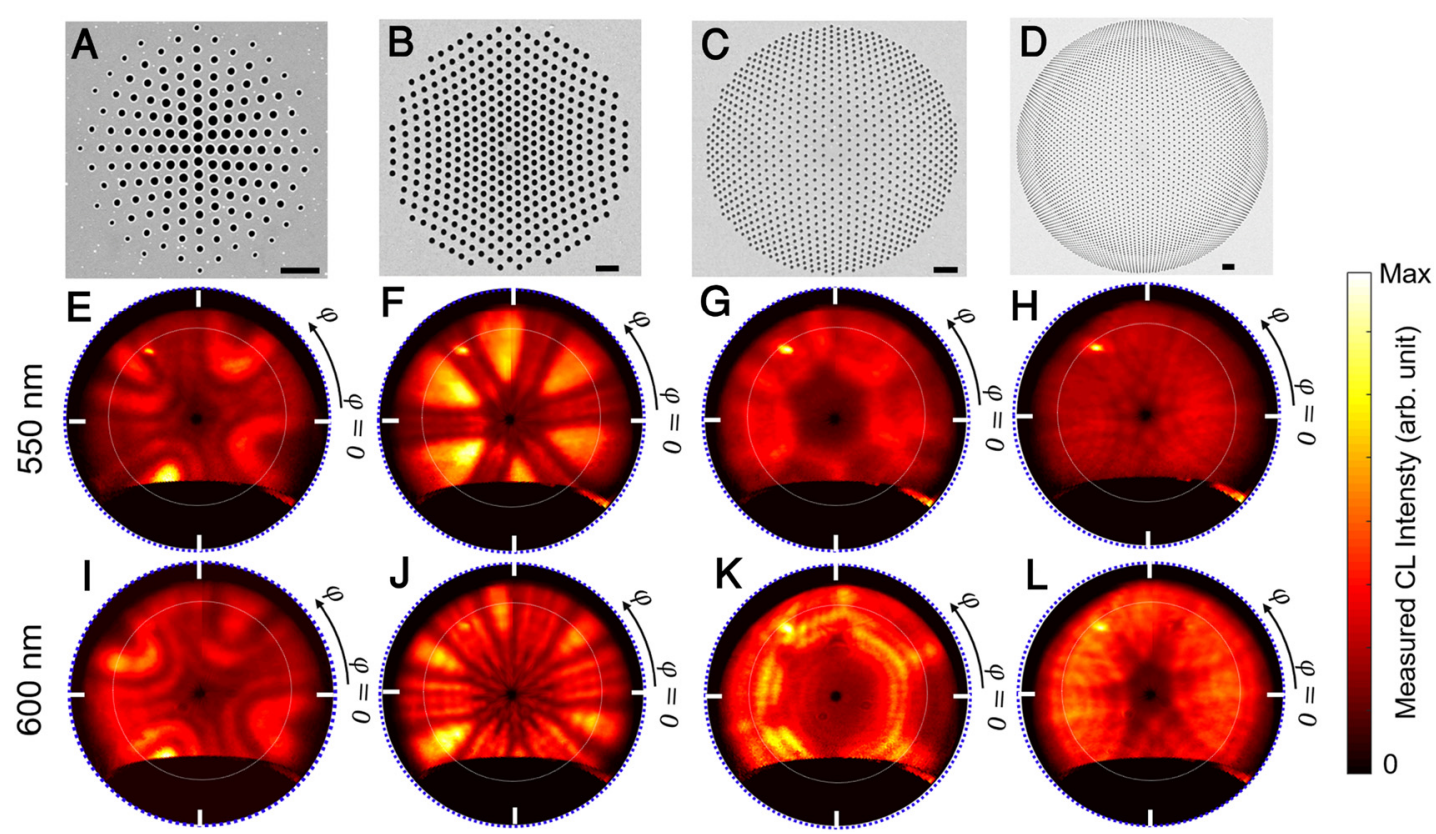

Figure 11: (A-D) Scanning electron microscopy images of different fabricated photon sieves. (E-H) Far-field angle-resolved CL maps at $550 \mathrm{~nm}$ wavelength with $50 \mathrm{~nm}$ bandpass filter from (A-D) photon sieves, respectively. (I-L) Angle-resolved CL maps at $600 \mathrm{~nm}$ wavelength with $50 \mathrm{~nm}$ bandpass filter from (A-D) photon sieves, respectively. The inner (white) and outer (blue) circles in images (E-L) represent the $\theta=60^{\circ}$ and $\theta=90^{\circ}$ planes, respectively. The scale bar in SEM images is $1 \mu \mathrm{m}$. The incident electron beam is probed very near to the center of each photon sieves. $\mathrm{CL}$, cathodoluminescence. 

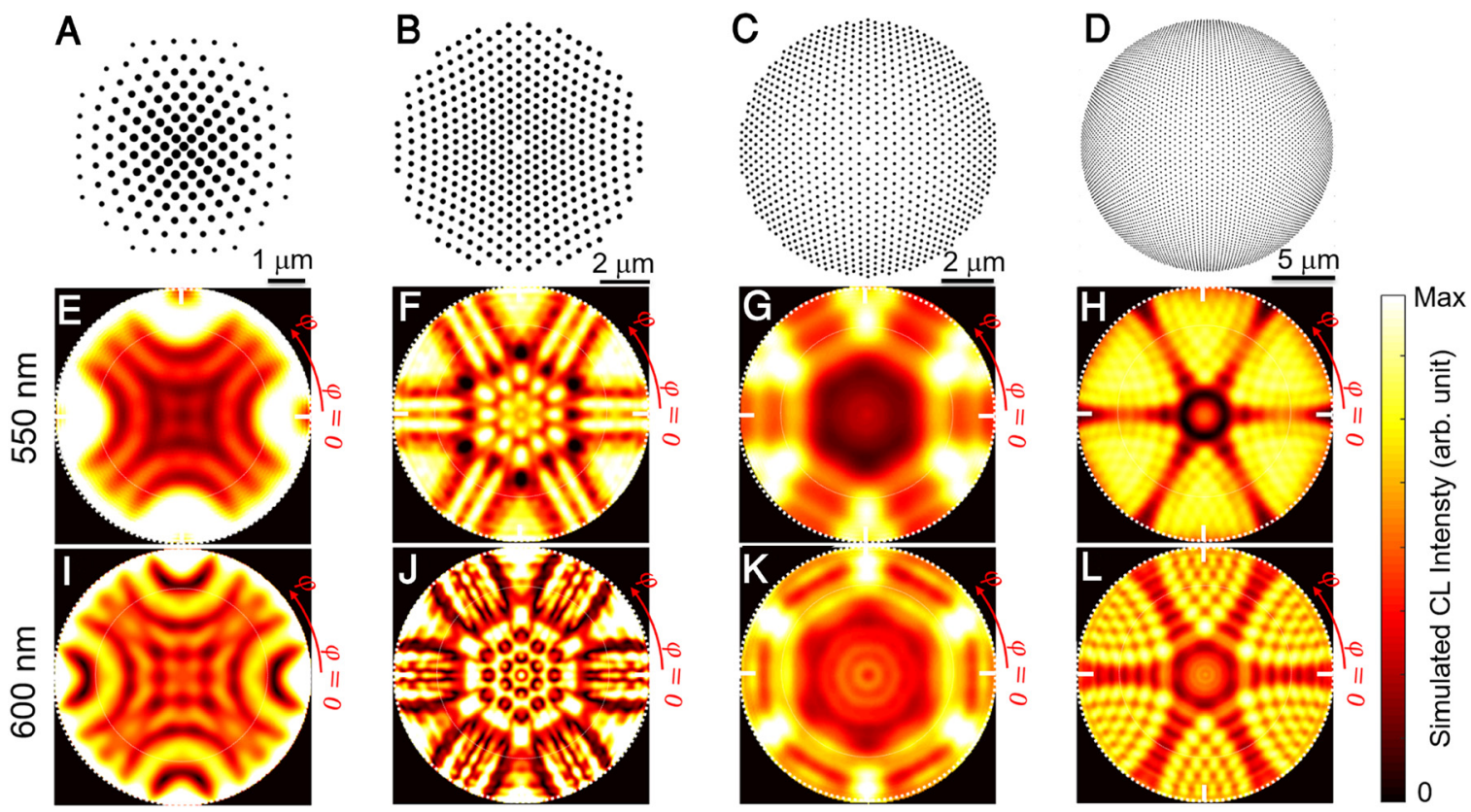

Figure 12: (A-D) Masks used in our analytical derivations to calculate the $C L$ maps. (E-H) Far-field angle-resolved $C L$ maps at $550 \mathrm{~nm}$ wavelength with $50 \mathrm{~nm}$ bandpass filter from (A-D) photon sieves, respectively, and (I-L) angle-resolved $C L$ maps at $600 \mathrm{~nm}$ wavelength with $50 \mathrm{~nm}$ bandpass filter from (A-D) photon sieves, respectively. $C L$, cathodoluminescence.

tailoring the radiation properties of photon sieves can be gained with a careful choice of electron impact position. Figure 13 shows angle-resolved CL maps from the first photon sieve structure versus the electron impact position. The direction of the main lobe in the radiation pattern is triggered by positioning the electron near to one of the 4 quadrants in our fourfold symmetry structure. Thus, the directionality of the emission can be tailored as well. Moreover, the emission spectrum demonstrates a broad bandwidth of $350 \mathrm{~nm}$ (at full-wave half maximum intensity), whereas the center wavelength is $710 \mathrm{~nm}$.

\section{Outlook for spectral interferometry}

When an electron interacts with a nanostructure or a thin film, ultrashort pulses in the attoseconds order are emitted due to the short interaction time of the electron with the material. Characterization of ultrashort pulses is important for understanding rich physics dynamics on the nanoscale $[180,181]$. The properties of extremely short emitted pulses can only be retrieved via ultrafast pulse characterization techniques such as spectral interferometry since Fourierbased spectroscopy techniques are not sufficient for ultrashort pulse analysis. In a spectral interferometry apparatus, two optical pulses with a certain delay are superimposed and the intensity of the superposition is captured in the spectral domain, demonstrating typical spectral fringes. The interference principle can be used for retrieving the spectral phase, similar to the spatial holography techniques retrieving spatial phases. Hence, timeresolved spectroscopy techniques that rely on electrons as a probe source meet the demand for retrieving the spatiotemporal profile of the CL emissions from the sample [181, 182].

In addition, electron microscopes with pulsed electron sources form the framework of ultrafast techniques, thanks to subpicosecond time resolution and angstrom spatial resolution. Ultrafast microscopes normally use photoemission electron sources interacting with pulsed laser excitations. In a pump-probe configuration, the photoinduced excitations of the sample are probed with electron pulses; hence, characterizing the sample excitations in the time-energy phase space is made feasible [183, 184]. Although the use of subpicosecond pulses to probe a sample via the electron microscope paves the way for ultrafast analysis, the dynamics of a material in the attoseconds range have not yet been reported. This is due to a lack of perfect synchronization between the arrival times of the electron to the probed sample and the laser time reference. It is important to add that despite the apparent advantages of electron-based time-energy characterization techniques, methods such as spectral interferometry to 


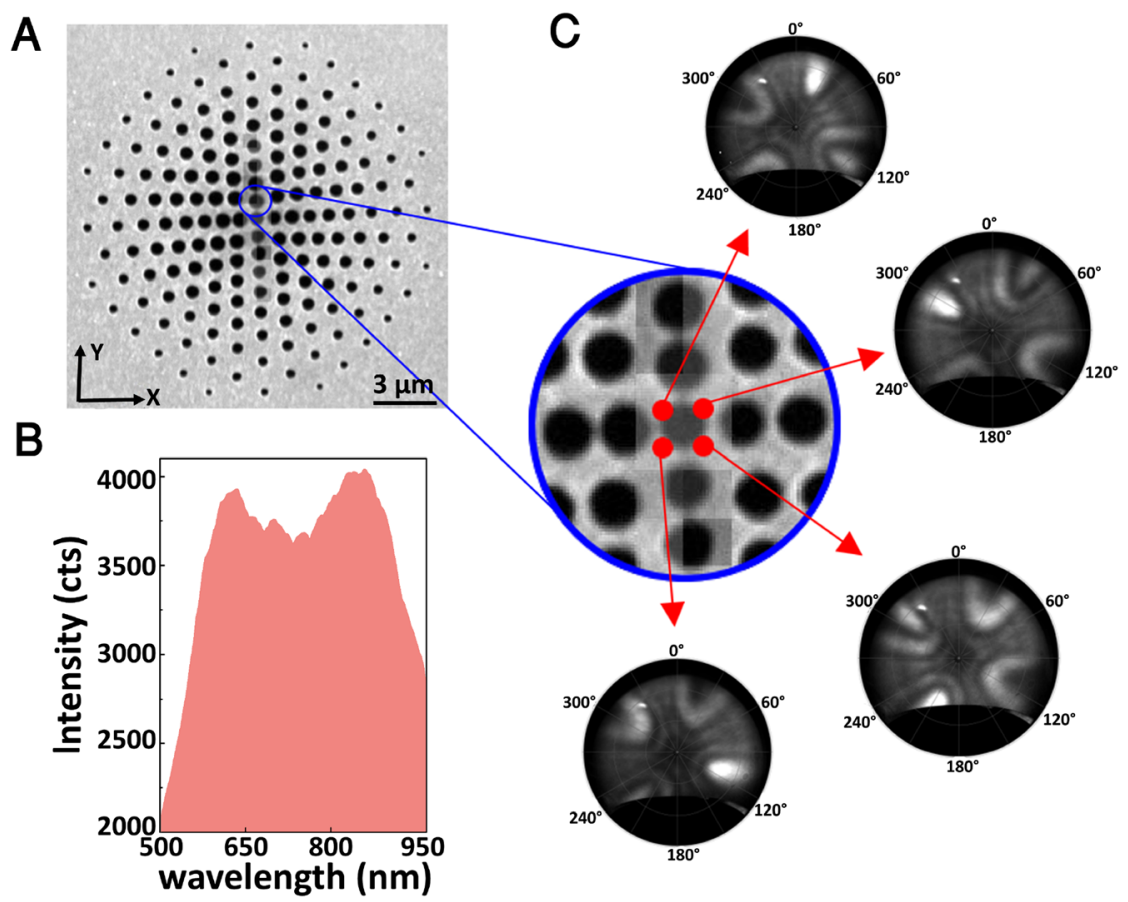

Figure 13: (A) Angle-resolved CL maps from the structure versus the electron impact position. (B) Integrated spectrum for the scanned region involving the central nanopinhole. $\mathrm{CL}$, cathodoluminescence. provide access for the phase data have not yet been reported. Recently, a system for characterizing the CL emission within the time-energy space has been proposed [16]. This system comprises an EDPHS with tailored emission properties and a manipulator as the only additional component to an electron microscope and allows for timeresolved spectroscopy and retrieving spectral phase of the sample excitations, without the need for an additional laser source. A relativistic electron traversing a carefully engineered metamaterial can radiate coherent optical beams as discussed in the previous chapter. A mesoscopic metamaterial-based EDPHS has been designed to generate coherent and focused TR along the electron trajectory [16] (Figure 14A). For the design of an efficient EDPHS for spectroscopic applications, the following fundamental conditions are to be considered. First, the EDPHS should be able to couple the electron-induced polarization to the farfield radiation. Second, the EDPHS should exhibit focused radiation on the sample. Third, the EDPHS should manifest a broadband far-field spectrum to investigate the ultrafast responses of the sample with high efficiency. The multilayer metal/dielectric structure in an effective medium approximation shows an electromagnetic hyperbolic behavior (Figure 14A). This behavior results in a huge photonic density of state and an enhanced coupling efficiency of the quantum emitters to the optical modes. To meet the already stated conditions for an effective design of EDPHSs, void hexagonal photonic crystals were incorporated into the structure. The collective radiation from the photonic crystal elements leads to an efficient coupling to the radiation continuum. Therefore, nonradiative decay channels are avoided. A sample positioned along the electron trajectory will interact with both the electron and the EDPHS excitations.

The EDPHS excitation interferes with the electroninduced CL emission of the sample, either destructively or constructively, depending on the distance $\mathrm{L}$ between the sample and EDPHS. The sample is positioned at the focal point of the EDPHS, and its distance from the EDPHS is precisely tuned (Figure 14B). Two samples, Ag disc and Si disc, with $300 \mathrm{~nm}$ and $40 \mathrm{~nm}$ diameter, respectively, were used to investigate the functionality of this method. Here, we discuss more the Ag disc sample under the influence of the proposed EDPHS. Figure 14C shows the CL spectra for the Ag sample and the EDPHS structure by gray and green shadow regions, respectively. The CL emissions from the samples are intense, and a clear peak is observed at $E=2.9 \mathrm{eV}$ due to excitations of plasmons. For the case where the Ag sample is positioned along both the EDPHS and the electron trajectory, they form a coupled structure with the overall CL spectrum which is not just the sum of the CL spectrum of the different elements. To retrieve this spectral phase, a referenced signal is required which is taken as the emission from the EDPHS. From the Fourier transform spectral interferometry [181], one single CL spectrum of the coupled EDPHS/sample system at a pulse/ 
A

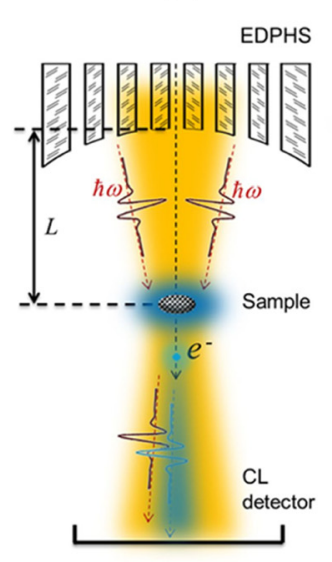

B

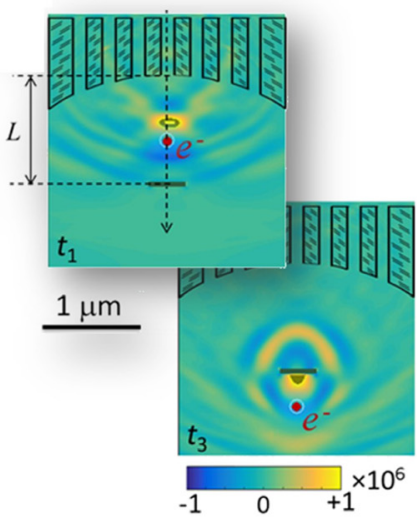

C

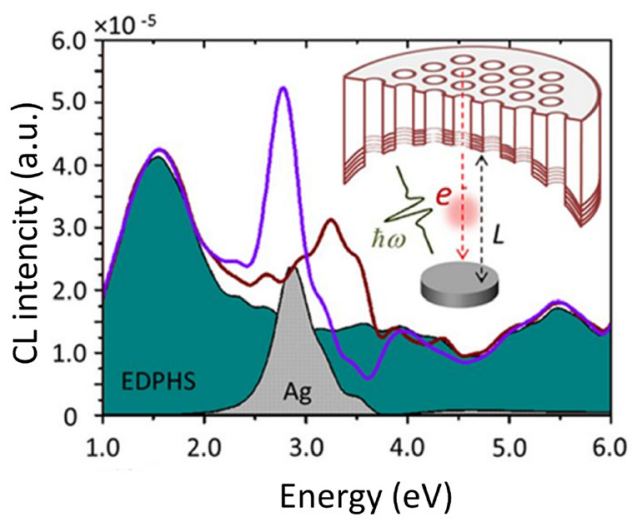

Figure 14: (A) The setup is composed of a simulated EDPHS, which on interaction with a swift electron generates photons that are scattered in a unidirectional way toward the sample and focused on it. (B) The EDPHS excitation interferes with the electron-induced CL emission of the sample, either destructively or constructively, depending on the distance $L$ between the sample and EDPHS. The sample is positioned at the focal point of the EDPHS. (C) CL spectra for an isolated EDPHS (teal shadowed region) and also a sample coupled to the EDPHS at distances $L=1.0 \mu \mathrm{m}$ between the sample and EDPHS for silver disc [16]. CL, cathodoluminescence; EDPHS, electron-driven photon source.

delay would be enough to retrieve the spectral pulse. However, the use of the full energy distance map allows for better noise reduction. The spatial profile of the generated light at the focal point depends very much on the design principle. For example, for structured light discussed in Section 3.4 (Figure 12), the pulse width at the focus is about the wavelength of the generated light. The EDPHS, when manipulated and engineered appropriately, can serve as a unique source for probing nanoscale properties of nanomaterials.

The advantage of using an EDPHS instead of a laser excitation is to avoid the requirements for intensively manipulating an electron microscope configuration for the insertion of optics and laser excitations. EDPHSs can be designed as well to achieve tailored light properties with specified polarization and angular momentum states [186]. Thanks to the various possibilities existing for nanofabrication tools, three-dimensional configurations, multilayered geometries, as well as lateral patterning using focused ion beam, and electron beam lithography can be applied for further tailoring the emission properties of EDPHSs. However, reaching arbitrary high photon numbers is seemingly challenging and requires further infrastructures utilizing other radiation mechanisms such as Larmor radiation and free electron lasers. An additional obvious challenge is to precisely adjusting the lateral and longitudinal positioning of sample and EDPHS structures. Indeed, nanopositioners and nanorobotics tools for electron microscopes incorporating piezoelectric effects are well advanced and could be used for the idea discussed here as well [186].

\section{Method}

\subsection{Fabrication of the electron-driven photon source}

Structures discussed in this work were fabricated by deposition of 40 to $50 \mathrm{~nm}$ gold films onto the $20 \mathrm{~nm} \mathrm{Si}_{3} \mathrm{~N}_{4}$ transmission electron microscopy membranes. To secure the membrane fragile structure, all the holes were created by direct milling with doubly charged $\mathrm{Au}^{++}$ions. The beam acceleration voltage was adjusted to $35 \mathrm{kV}$, and the Raith ionLine Plus system (a dedicated high-resolution ion beam structuring tool) was utilized to accomplish the high-resolution outcome.

\subsection{Cathodoluminescence}

The optical detection system used for the CL measurements was provided by Delmic SPARC. Measurements presented in Figures 11 and 12 were conducted in a CL compartment attached to a Thermo Fisher Quanta650 SEM ${ }^{3}$ in AMOLF, and data shown in Figure 13 were collected using Delmic SPARC CL detector installed on a Zeiss SIGMA field emission microscope in the Christian Albrechts University in Kiel. The acceleration voltage for all measurements was $30 \mathrm{keV}$, and light collection was performed by an off-axis paraboloid mirror mounted in an adjustable micromanipulation system. The mirror had an acceptance angle of $1.46 \pi \mathrm{sr}$, a focal distance of $0.5 \mathrm{~mm}$, and a $600 \mu \mathrm{m}$ diameter hole above the focal point for supporting the electron excitation.

\subsection{Simulations}

Electron-induced field simulations were performed using a semiclassical approach in which the electron is modeled by a current

3 https://www.fei.com/products/sem/quanta-sem/\#gsc.tab=0. 
density distribution associated with a moving charge at a certain kinetic energy ( $E=30 \mathrm{kV}$ in this work). Finite-difference time-domain simulations with a mesh size of $2 \mathrm{~nm}$ were performed by applying a self-developed code for a deeper perception of the temporal distribution of the generated electron-induced radiation. The permittivity of the gold was modeled using a Drude model in addition to two critical point functions [187, 188]. Absorbing boundary conditions were introduced to satisfy the radiation boundary conditions.

\section{Conclusion and future scope}

Mechanisms of radiation from electron beams interacting with the nanostructures have been thoroughly outlined, and new efforts toward harnessing the radiation via miniaturized light sources have been highlighted here. We particularly outlined the possibilities of using different nanofabrication concepts routinely used in nanooptics to tailor the electron-induced radiation. Smith-Purcell effect, perhaps the most widely used concept, has been already raised to the emergence of powerful $\mathrm{THz}$ sources, known as orotrons. In addition, metamaterial sources, holographic sources, and photon sieves were discussed. We have shown that the photon sieves, being fabrication-wise more straight forward, have the potential of realizing sources for arbitrary shaped light beams. A photon sieve can be structured to tailor material properties effectively to mimic a curved geometry or to realize a diffractive lens element as shown here. However, all concepts besides the SmithPurcell effect are mostly harnessing the transversal light patterns, whereas the longitudinal degree of freedom is only indirectly touched. Combining the transversal and longitudinal degrees of freedom to propose 3-dimensional light sources, though fabrication-wise can be challenging, could offer a multitude of possibilities in coherently manipulating not only the light shapes but also its intensity. Moreover, there exists plenty of room in investigating the role of materials stacking, particularly with the advent of van der Waals quantum materials, to tailor further the radiation properties toward the generation of vortex sources, for example, using valley degrees of freedoms in quantum materials. On the spectral interferometry side, a precise tuning of the lateral and longitudinal distances between the sample and the EDPHS can be challenging due to temporal vibrations and radiation pressure. Nevertheless, conceptually the idea has the potential to explore various quantum mechanical fundamental concepts such as the role of incoherent and coherent relaxation rates in different materials, retrieving the density matrix of the material excitations such as plasmons and excitons, as well as investigating the roles of a partial collapse of the wave function and decoherence in open versus closed quantum mechanical systems, though all yet to be explored.

Acknowledgment: This project has received funding from the European Research Council (ERC) under the European Union's Horizon 2020 research and innovation program, grants agreements No. 802130 (Kiel, Nanobeam) and Stuttgart, ComplexPlas. MH and HG acknowledge financial support from the Bundesministerium für Bildung und Forschung, Deutsche Forschungsgemeinschaft (SPP1839), and Baden-Württemberg Stiftung.

Author contribution: All the authors have accepted responsibility for the entire content of this submitted manuscript and approved submission.

Research funding: This project has received funding from the European Research Council (ERC) under the European Union's Horizon 2020 research and innovation program, grants agreements No. 802130, Bundesministerium für Bildung und Forschung, Deutsche Forschungsgemeinschaft (SPP1839), and Baden-Württemberg Stiftung.

Conflict of interest statement: The authors declare no conflicts of interest regarding this article.

\section{References}

[1] W. Crookes, "XVI. Contributions to molecular physics in high vacua. Magnetic deflection of molecular trajectory.-Laws of magnetic rotation in high and low vacua.-Phosphorogenic properties of molecular discharge," Phil. Trans. R. Soc. Lond., vol. 170, pp. 641-662, 1879.

[2] B. Falkenburg and F. Weinert, "Indeterminism and determinism in quantum mechanics," in Compendium of Quantum Physics, Berlin, Heidelberg, Springer, 2009, pp. 307-311.

[3] P. L. Gould, G. A. Ruff, and D. E. Pritchard, "Diffraction of atoms by light: the near-resonant Kapitza-Dirac effect," Phys. Rev. Lett., vol. 56, no. 8, p. 827, 1986.

[4] D. L. Freidmund, K. Aflatooni, and H. Batelaan, "Observation of the Kapitza-Dirac effect,” Nature, vol. 413, no. 6852, pp. 142-143, 2001.

[5] A. Feist, K. E. Echternkamp, J. Schauss, S. V. Yalunin, S. Schafer, and C. Ropers, "Quantum coherent optical phase modulation in an ultrafast transmission electron microscope," Nature, vol. 521, no. 7551, pp. 200-203, 2015.

[6] N. Talebi, "Electron-light interactions beyond the adiabatic approximation: recoil engineering and spectral interferometry," Adv. Phys. X, vol. 3, no. 1, p. 1499438, 2018.

[7] I. Madan, G. M. Vanacore, E. Pomarico, et al., "Holographic imaging of electromagnetic fields via electron-light quantum interference," Sci. Adv., vol. 5, no. 5, p. eaav8358, 2019.

[8] N. Talebi, W. Sigle, R. Vogelgesang, and P. van Aken, "Numerical simulations of interference effects in photon-assisted electron energy-loss spectroscopy," New J. Phys., vol. 15, no. 5, p. 053013 , 2013. 
[9] O. Kfir, H. Lourenco-Martins, G. Storeck, M. Sivis, T. R. Harvey, and T.J. Kippenberg, "Controlling free electrons with optical whisperinggallery modes,” Nature, vol. 582, no. 7810, pp. 46-49, 2020.

[10] N. Talebi, "Strong interaction of slow electrons with near-field light visited from first principles," Phys. Rev. Lett., vol. 125, pp. 080401, 2020.

[11] B. Barwick, D. J. Flannigan, and A. H. Zewail, "Photon-induced near-field electron microscopy,” Nature, vol. 462, no. 7275 , pp. 902-906, 2009.

[12] A. Losquin, L. F. Zagonel, V. Myroshnychenko, et al., "Unveiling nanometer scale extinction and scattering phenomena through combined electron energy loss spectroscopy and cathodoluminescence measurements," Nano Lett., vol. 15, no. 2, pp. 1229-1237, 2015.

[13] F. G. De Abajo, "Optical excitations in electron microscopy," Rev. Mod. Phys., vol. 82, no. 1, p. 209, 2010.

[14] A. Polman, M. Kociak, and F. J. de Abajo, "Electron-beam spectroscopy for nanophotonics," Nat. Mater., vol. 15, p. 1, 2019.

[15] X. Bendana, A. Polman, and F. J. Garcia de Abajo, "Single-photon generation by electron beams," Nano Lett., vol. 11, no. 12, pp. 5099-5103, 2011.

[16] N. Talebi, "Spectral interferometry with electron microscopes," Sci. Rep., vol. 6, no. 1, pp. 1-0, 2016.

[17] U. Happek, A. J. Sievers, and E. B. Blum, "Observation of coherent transition radiation,” Phys. Rev. Lett., vol. 67, no. 21, p. 2962, 1991.

[18] P. A. Cherenkov, "Visible emission of clean liquids by action of $y$ radiation," InDokl. Akad. Nauk SSSR, vol. 2, no. 8, pp. 451-454, 1934.

[19] J. H. Brownell, G. Doucas, M. F. Kimmitt, J. H. Mulvey, M. Omori, and J. E. Walsh, "The angular distribution of the power produced by Smith-Purcell radiation,” J. Phys. Appl. Phys., vol. 30, no. 17, p. 2478, 1997.

[20] H. Bethe and W. Heitler, "On the stopping of fast particles and on the creation of positive electrons," Proc. R. Soc. Lond. Ser. A Contain. Pap. A Math. Phys. Character, vol. 146, no. 856, pp. 83-112, 1934.

[21] A. Gover, P. Dvorkis, and U. Elisha, "Angular radiation pattern of Smith-Purcell radiation,” JOSA B, vol. 1, no. 5, pp. 723-728, 1984.

[22] S. E. Korbly, A. S. Kesar, J. R. Sirigiri, and R. J. Temkin, "Observation of frequency-locked coherent terahertz SmithPurcell radiation," Phys. Rev. Lett., vol. 94, no. 5, p. 054803, 2005.

[23] G. Kube, H. Backe, H. Euteneuer, et al., "Observation of optical Smith-Purcell radiation at an electron beam energy of $855 \mathrm{MeV}$," Phys. Rev. E, vol. 65, no. 5, p. 056501, 2002.

[24] G. Doucas, J. H. Mulvey, M. Omori, J. Walsh, and M. F. Kimmitt, "First observation of Smith-Purcell radiation from relativistic electrons," Phys. Rev. Lett., vol. 69, no. 12, p. 1761, 1992.

[25] B. J. Brenny, T. Coenen, and A. Polman, "Quantifying coherent and incoherent cathodoluminescence in semiconductors and metals," J. Appl. Phys., vol. 115, no. 24, p. 244307, June 282014.

[26] W. L. Barnes, A. Dereux, and T. W. Ebbesen, "Surface plasmon subwavelength optics," Nature, vol. 424, no. 6950, pp. 824830, Aug. 2003.

[27] M. V. Bashevoy, F. Jonsson, A. V. Krasavin, N. I. Zheludev, Y. Chen, and M. I. Stockman, "Generation of traveling surface plasmon waves by free-electron impact," Nano Lett., vol. 6, no. 6, pp. 1113-1115, 2006.
[28] D. Spirkoska, J. Arbiol, A. Gustafsson, et al., "Structural and optical properties of high quality zinc-blende/wurtzite GaAs nanowire heterostructures," Phys. Rev. B, vol. 80, no. 24, p. 245325, 2009.

[29] L. H. Tizei and M. Kociak, "Spatially resolved quantum nanooptics of single photons using an electron microscope," Phys. Rev. Lett., vol. 110, no. 15, p. 153604, 2013.

[30] C. W. Chen, K. H. Chen, C. H. Shen, et al., "Anomalous blueshift in emission spectra of $\mathrm{ZnO}$ nanorods with sizes beyond quantum confinement regime," Appl. Phys. Lett., vol. 88, no. 24, p. 241905, 2006.

[31] Z. Mahfoud, A. T. Dijksman, C. Javaux, et al., "Cathodoluminescence in a scanning transmission electron microscope: a nanometer-scale counterpart of photoluminescence for the study of ii-vi quantum dots," J. Phys. Chem., vol. 4, no. 23, pp. 4090-4094, 2013.

[32] S. I. Vavilov, "On the possible causes of blue y-glow of liquids," InCR Dokl Akad Nauk SSSR, vol. 2, no. 8, p. 457, 1934.

[33] I. E. Tamm and I. M. Frank, "Coherent radiation of fast electrons in a medium," InDokl. Akad. Nauk SSSR, vol. 14, no. 3, pp. 107-112, 1937.

[34] O. Heaviside, "The electro-magnetic effects of a moving charge," The Electrician, vol. 22, pp. 147-148, 1888.

[35] O. M. Bilaniuk, “Tachyons," J. Phys. Conf. Ser., vol. 196, no. 1, p. $012021,2009$.

[36] Z. Duan, X. Tang, Z. Wang, et al., "Observation of the reversed Cherenkov radiation,” Nat. Commun., vol. 8, no. 1, pp. 1-7, 2017.

[37] V. G. Veselago, "The electrodynamics of substances with simultaneously negative values of Img Align= Absmiddle Alt $=\varepsilon$ Eps/Img and $\mu$," Physics-Uspekhi, vol. 10, no. 4, pp. 509-514, 1968.

[38] Z. Duan, B. I. Wu, J. Lu, J. A. Kong, and M. Chen, "Reversed Cherenkov radiation in unbounded anisotropic double-negative metamaterials," J. Phys. D Appl. Phys., vol. 42, no. 18, p. 185102, 2009.

[39] C. Luo, M. Ibanescu, S. G. Johnson, and J. D. Joannopoulos, "Cerenkov radiation in photonic crystals," Science, vol. 299, no. 5605, pp. 368-371, 2003.

[40] J. Lu, T. M. Grzegorczyk, Y. Zhang, et al., "Čerenkov radiation in materials with negative permittivity and permeability," Opt. Express, vol. 11, no. 7, pp. 723-734, 2003.

[41] Z. Duan, B. I. Wu, J. Lu, J. Au Kong, and M. Chen, "Reversed Cherenkov radiation in a waveguide filled with anisotropic double-negative metamaterials," J. Appl. Phys., vol. 104, no. 6, p. 063303, 2008.

[42] C. V. Festenberg, "Energy-loss measurements on III-V compounds," Univ. Hamburg, vol. 227, pp. 453-481, 1969.

[43] C. H. Chen, J. Silcox, and R. Vincent, "Electron-energy losses in silicon: bulk and surface plasmons and Čerenkov radiation," Phys. Rev. B, vol. 12, no. 1, p. 64, 1975.

[44] N. Yamamoto, F. J. de Abajo, and V. Myroshnychenko, "Interference of surface plasmons and Smith-Purcell emission probed by angle-resolved cathodoluminescence spectroscopy," Phys. Rev. B, vol. 91, no. 12, p. 125144, 2015.

[45] T. Coenen, E. J. Vesseur, A. Polman, and A. F. Koenderink, "Directional emission from plasmonic Yagi-Uda antennas probed by angle-resolved cathodoluminescence spectroscopy," Nano Lett., vol. 11, no. 9, pp. 3779-3784, 2011.

[46] R. Sapienza, T. Coenen, J. Renger, M. Kuttge, N. F. Van Hulst, and A. Polman, "Deep-subwavelength imaging of the modal 
dispersion of light," Nat. Mater., vol. 11, no. 9, pp. 781-787, 2012.

[47] K. Korzeb, M. Gajc, and D. A. Pawlak, "Compendium of natural hyperbolic materials," Opt. Express, vol. 23, no. 20, pp. 2540625424, 2015.

[48] S. Misra, L. Li, D. Zhang, et al., "Self-assembled ordered threephase $\mathrm{Au}-\mathrm{BaTiO3-ZnO}$ vertically aligned nanocomposites achieved by a templating method," Adv. Mater., vol. 31, no. 7, p. 1806529, 2019.

[49] I. I. Smolyaninov, and A. V. Kildishev, "Light propagation through random hyperbolic media," Opt. Lett., vol. 38, no. 6, pp. 971-973, 2013.

[50] P. Shekhar, S. Pendharker, D. Vick, M. Malac, and Z. Jacob, “Fast electrons interacting with a natural hyperbolic medium: bismuth telluride," Opt. Express, vol. 27, no. 5, pp. 6970-6975, 2019.

[51] M. Esslinger, R. Vogelgesang, N. Talebi, et al., "Tetradymites as natural hyperbolic materials for the near-infrared to visible," ACS Photonics, vol. 1, no. 12, pp. 1285-1289, 2014

[52] N. Talebi, "Interaction of electron beams with optical nanostructures and metamaterials: from coherent photon sources towards shaping the wave function,"J. Opt., vol. 19, no. 10, p. 103001, 2017

[53] J. D. Caldwell, A. V. Kretinin, Y. Chen, et al., "Sub-diffractional volume-confined polaritons in the natural hyperbolic material hexagonal boron nitride," Nat. Commun., vol. 5, no. 1, pp. 1-9, 2014.

[54] N. Talebi, "Near-field-mediated photon-electron interactions," Springer Ser. Opt. Sci., vol. 228, pp. 31-52, 2019.

[55] J. Tao, L. Wu, G. Zheng, and S. Yu, "Cherenkov polaritonic radiation in a natural hyperbolic material," Carbon, vol. 150, pp. 136-141, 2019.

[56] F. S. Hage, R. J. Nicholls, J. R. Yates, et al., "Nanoscale momentum-resolved vibrational spectroscopy," Sci. Adv., vol. 4, no. 6, p. eaar7495, 2018

[57] F. Liu, L. Xiao, Y. Ye, et al., “Integrated Cherenkov radiation emitter eliminating the electron velocity threshold," Nat. Photonics, vol. 11, no. 5, p. 289, 2017.

[58] V. L. Ginzburg, and I. M. Frank, "Radiation of a uniformly moving electron due to its transition from one medium into another," $Z h$. Eksp. Teor. Fiz., vol. 9, pp. 353-362, 1945.

[59] J. Zheng, K. A. Tanaka, T. Miyakoshi, et al., "Theoretical study of transition radiation from hot electrons generated in the lasersolid interaction," Phys. Plasmas, vol. 10, no. 7, pp. 2994-3003, 2003.

[60] L. Wartski, S. Roland, J. Lasalle, M. Bolore, and G. Filippi, "Interference phenomenon in optical transition radiation and its application to particle beam diagnostics and multiple-scattering measurements,"J. Appl. Phys., vol. 46, no. 8, pp. 3644-3653, 1975.

[61] B. Dolgoshein, "Transition radiation detectors," Nucl. Instrum. Methods Phys. Res. Sect. A Accel. Spectrom. Detect. Assoc. Equip., vol. 326, no. 3, pp. 434-469, 1993.

[62] V. L. Ginzburg, "Transition radiation and transition scattering," Phys. Scripta, vol. 1982, no. T2A, p. 182, 1982.

[63] V. L. Ginzburg and V. N. Tsytovich, "Several problems of the theory of transition radiation and transition scattering," Phys. Rep., vol. 49, no. 1, pp. 1-89, 1979.

[64] E. Kröger, "Calculation of the energy losses of fast electrons in thin foils with retardation," Univ. Hamburg, vol. 216 pp. 115-135, 1968.
[65] F. G. De Abajo, A. Rivacoba, N. Zabala, and N. Yamamoto, "Boundary effects in Cherenkov radiation," Phys. Rev. B, vol. 69 , no. 15, p. 155420, 2004.

[66] N. Talebi, S. Meuret, S. Guo, et al., "Merging transformation optics with electron-driven photon sources," Nat. Commun., vol. 10, no. 1, pp. 1-8, 2019.

[67] N. Papasimakis, V. A. Fedotov, Y. H. Fu, D. P. Tsai, and N. I. Zheludev, "Coherent and incoherent metamaterials and order-disorder transitions," Phys. Rev. B, vol. 80, no. 4, p. 041102, 2009.

[68] E. Ozbay, "Plasmonics: merging photonics and electronics at nanoscale dimensions," Science, vol. 311, no. 5758, pp. 189193, 2006

[69] Y. A. Akimov and H. S. Chu, "Plasmon-plasmon interaction: controlling light at nanoscale," Nanotechnology, vol. 23, no. 44 p. 444004, 2012

[70] J. M. Pitarke, V. M. Silkin, E. V. Chulkov, and P. M. Echenique, "Theory of surface plasmons and surface-plasmon polaritons," Rep. Prog. Phys., vol. 70, no. 1, p. 1, 2006.

[71] R. H. Ritchie, "Plasma losses by fast electrons in thin films," Phys. Rev., vol. 106, no. 5, p. 874, 1957.

[72] C. J. Powell and J. B. Swan, "Origin of the characteristic electron energy losses in aluminum,” Phys. Rev., vol. 115, no. 4, p. 869, 1959.

[73] C. J. Powell and J. B. Swan, "Origin of the characteristic electron energy losses in magnesium," Phys. Rev., vol. 116, no. 1, p. 81, 1959.

[74] P. E. Batson, "Surface plasmon coupling in clusters of small spheres," Phys. Rev. Lett., vol. 49, no. 13, p. 936, 1982.

[75] D. Ugarte, C. Colliex, and P. Trebbia, "Surface-Plasmon and interface-plasmon modes on small semiconducting spheres," Phys. Rev. B, vol. 45, p. 4332, 1992.

[76] J. Nelayah, M. Kociak, O. Stephan, et al., "Mapping surface plasmons on a single metallic nanoparticle," Nat. Phys., vol. 3, no. 5, pp. 348-353, 2007

[77] V. Bastys, I. Pastoriza-Santos, B. Rodríguez-González, R. Vaisnoras, and L. M. Liz-Marzán, "Formation of silver nanoprisms with surface plasmons at communication wavelengths," Adv. Funct. Mater., vol. 16, no. 6, pp. 766-773, 2006.

[78] C. Clavero, "Plasmon-induced hot-electron generation at nanoparticle/metal-oxide interfaces for photovoltaic and photocatalytic devices," Nat. Photonics, vol. 8, no. 2, p. 95, 2014.

[79] N. Yamamoto, "Cathodoluminescence of surface plasmon induced light emission," in Transmission Electron Microscope, London, UK: Intech Open, 2010, https://doi.org/10.5772/35244.

[80] T. Sannomiya, A. Konečná, T. Matsukata, et al., "Cathodoluminescence phase extraction of the coupling between nanoparticles and surface plasmons polaritons," Nano Lett., vol. 1, no. 20, pp. 592-598, 2019.

[81] N. J. Schilder, H. Agrawal, E. C. Garnett, and A. Polman, "Phaseresolved surface plasmon scattering probed by cathodoluminescence holography," ACS Photonics, vol. 7, no. 6 , p. 1476, 1957.

[82] R. de Waele, A. F. Koenderink, and A. Polman, "Tunable nanoscale localization of energy on plasmon particle arrays," Nano Lett., vol. 7, no. 7, pp. 2004-2008, 2007.

[83] J. Aizpurua, P. Hanarp, D. S. Sutherland, M. Käll, G. W. Bryant, and F. G. De Abajo, “Optical properties of gold nanorings," Phys. Rev. Lett., vol. 90, no. 5, p. 057401, 2003. 
[84] J. Aizpurua, G. W. Bryant, L. J. Richter, F. G. De Abajo, B. K. Kelley, and T. Mallouk, "Optical properties of coupled metallic nanorods for field-enhanced spectroscopy,” Phys. Rev. B, vol. 71, no. 23, p. 235420, 2005.

[85] M. Bosman, V. J. Keast, M. Watanabe, A. I. Maaroof, and M. B. Cortie, "Mapping surface plasmons at the nanometre scale with an electron beam," Nanotechnology, vol. 18, no. 16, p. $165505,2007$.

[86] K. Okamoto, I. Niki, A. Shvartser, Y. Narukawa, T. Mukai, and A. Scherer, "Surface-plasmon-enhanced light emitters based on InGaN quantum wells," Nat. Mater., vol. 3, no. 9, pp. 601-605, 2004.

[87] P. Mulvaney, "Surface plasmon spectroscopy of nanosized metal particles," Langmuir, vol. 12, no. 3, pp. 788-800, 1996.

[88] R. Sundararaman, P. Narang, A. S. Jermyn, W. A. Goddard III, and H. A. Atwater, "Theoretical predictions for hot-carrier generation from surface plasmon decay," Nat. Commun., vol. 5, no. 1, pp. 1-8, 2014.

[89] T. Holmgaard and S. I. Bozhevolnyi, "Theoretical analysis of dielectric-loaded surface plasmon-polariton waveguides," Phys. Rev. B, vol. 75, no. 24, p. 245405, 2007.

[90] L. Wu, H. S. Chu, W. S. Koh, and E. P. Li, "Highly sensitive graphene biosensors based on surface plasmon resonance," Opt. Express, vol. 18, no. 14, pp. 14395-14400, 2010.

[91] S. Guo, N. Talebi, A. Campos, et al., "Far-field radiation of threedimensional plasmonic gold tapers near apexes," ACS Photonics, vol. 6, no. 10, pp. 2509-2516, 2019.

[92] A. J. Haes and R. P. Duyne, "Preliminary studies and potential applications of localized surface plasmon resonance spectroscopy in medical diagnostics," Expert Rev. Mol. Diagn., vol. 4, no. 4, pp. 527-537, 2004.

[93] X. D. Hoa, A. G. Kirk, and M. Tabrizian, "Towards integrated and sensitive surface plasmon resonance biosensors: a review of recent progress," Biosens. Bioelectron., vol. 23, no. 2, pp. 151$160,2007$.

[94] J. B. Pendry, L. Martin-Moreno, and F. J. Garcia-Vidal, "Mimicking surface plasmons with structured surfaces," Science, vol. 305, no. 5685, pp. 847-848, 2004.

[95] N. Talebi and M. Shahabadi, "Spoof surface plasmons propagating along a periodically corrugated coaxial waveguide," J. Phys. D Appl. Phys., vol. 43, no. 13, p. 135302, 2010 Mar 18.

[96] C. Sönnichsen, B. M. Reinhard, J. Liphardt, and A. P. Alivisatos, "A molecular ruler based on plasmon coupling of single gold and silver nanoparticles," Nat. Biotechnol., vol. 23, no. 6, pp. 741745, 2005.

[97] D. Lazer, A. S. Pentland, L. Adamic, et al., "Life in the network: the coming age of computational social science," Science, vol. 323, no. 5915, pp. 721-723, 2009.

[98] F. Neubrech, T. Kolb, R. Lovrincic, et al., "Resonances of individual metal nanowires in the infrared," Appl. Phys. Lett., vol. 89, no. 25, p. 253104, 2006.

[99] G. W. Bryant, F. J. García de Abajo, and J. Aizpurua, "Mapping the plasmon resonances of metallic nanoantennas," Nano Lett., vol. 8, no. 2, pp. 631-636, 2008.

[100] G. R. Blumenthal and R. J. Gould, "Bremsstrahlung, synchrotron radiation, and compton scattering of high-energy electrons traversing dilute gases," Rev. Mod. Phys., vol. 42, no. 2, p. 237, 1970.
[101] C. A. Bertulani, D. T. De Paula, and V. G. Zelevinsky, "Bremsstrahlung radiation by a tunneling particle: a timedependent description," Phys. Rev. C, vol. 60, no. 3, p. 031602, 1999.

[102] M. Protopapas, D. G. Lappas, C. H. Keitel, and P. L. Knight, "Recollisions, bremsstrahlung, and attosecond pulses from intense laser fields," Phys. Rev., vol. 53, no. 5, p. R2933, 1996.

[103] J. Schwinger, W. Y. Tsai, and T. Erber, "Classical and quantum theory of synergic synchrotron-Čerenkov radiation," Ann. Phys., vol. 96, no. 2, pp. 303-332, 1976.

[104] E. S. Cramer, J. R. Dwyer, S. Arabshahi, I. B. Vodopiyanov, N. Liu, and H. K. Rassoul, "An analytical approach for calculating energy spectra of relativistic runaway electron avalanches in air," J. Geophys. Res. Space Phys., vol. 119, no. 9, pp. 77947823, 2014.

[105] A. Sandrock, S. R. Kelner, and W. Rhode, "Radiative corrections to the average bremsstrahlung energy loss of high-energy muons," Phys. Lett. B, vol. 776, pp. 350-354, 2018.

[106] G. D. Palazzi, "High-energy bremsstrahlung and electron pair production in thin crystals," Rev. Mod. Phys., vol. 40, no. 3, p. $611,1968$.

[107] U. Timm, "Coherent bremsstrahlung of electrons in crystals," Fortschr. Phys., vol. 17, no. 12, pp. 765-808, 1969.

[108] M. L. Ter-Mikaelian, High Energy Electromagnetic Processes in Condensed Media, New York, NY, Wiley, 1972, p. 466.

[109] B. Ferretti, "Sulla "Bremsstrahlung" nei cristalli," Il Nuovo Cimento (1943-1954), vol. 7, no. 2, pp. 118-134, 1950.

[110] L. Landau and I. Pomeranchuk, "Electron-cascade processes at very high energies," in US Atomic Energy Commission, Technical Information Service, vol. 92, 1953, p. 735.

[111] A. W. Saenz and H. Überall, "Theory of coherent bremsstrahlung," Radiat. Eff. Defect Solid, vol. 122, no. 2, pp. 401-416, 1991.

[112] H. Überall, "High-energy interference effect of bremsstrahlung and pair production in crystals," Phys. Rev., vol. 103, no. 4, p. 1055, 1956.

[113] H. Uberall, "Die Polarisation der quasi-monochromatischdien Bremsstrahlung von einem Einkristall," Z. Naturforsch. A., vol. 17, no. 4, pp. 332-334, 1962.

[114] I. J. Berson, "Multiphoton ionization and stimulated bremsstrahlung radiation in the case of short-range potentials," J. Phys. B Atom. Mol. Phys., vol. 8, no. 18, p. 3078, 1975.

[115] A. I. Akhiezer, P. I. Fomin, and N. F. Shul'Ga, "Coherent bremsstrahlung of electrons and positrons of ultrahigh energy in crystals," ZhETF Pisma Redaktsiiu, vol. 13, p. 713, 1971.

[116] A. A. Vorob'ev, N. I. Zimin, and S. A. Vorob'ev, "Radiation of relativistic electrons in axial channeling," Sov. Phys. J., vol. 20, no. 8, pp. 1112-1113, 1977.

[117] R. Lal and S. K. Joshi, "Coherent bremsstrahlung from relativistic channelled positrons," Pramana, vol. 15, no. 2, pp. 175-179, 1980.

[118] J. Lindhard, "Influence of crystal lattice on motion of energetic charged particles," Munksgaard, vol. 34, no. 16, 1965.

[119] W. Heitler, The Quantum Theory of Radiation, New York, USA: Dover Publication, 1984, pp. 5201-5283.

[120] J. U. Andersen, K. R. Eriksen, and E. Laegsgaard, “Planarchanneling radiation and coherent bremsstrahlung for $\mathrm{MeV}$ electrons," Phys. Scripta, vol. 24, no. 3, p. 588, 1981. 
[121] F. E. L. Undulators, C. S. O. L. Light, C. Bremsstrahlung, and X-rays, Parametric. Radiation by Relativistic Electrons, 1986.

[122] S. J. Smith and E. M. Purcell, "Visible light from localized surface charges moving across a grating," Phys. Rev., vol. 92, no. 4, p. 1069, 1953

[123] F. G. de Abajo, "Smith-Purcell radiation emission in aligned nanoparticles," Phys. Rev., vol. 61, no. 5, p. 5743, 1 May 2000.

[124] M. Kadic, T. Bückmann, R. Schittny, and M. Wegener, "Metamaterials beyond electromagnetism," Rep. Prog. Phys., vol. 76, no. 12, p. 126501, 2013.

[125] S. Zouhdi, A. Sihvola, and A. P. Vinogradov, Eds., Metamaterials and Plasmonics: Fundamentals, Modelling, Applications, Dordrecht, The Netherlands: Springer Science \& Business Media, 16 Dec. 2008.

[126] B. Luk'yanchuk, N. I. Zheludev, S. A. Maier, et al., "The Fano resonance in plasmonic nanostructures and metamaterials," Nat. Mater., vol. 9, no. 9, pp. 707-715, 2010.

[127] N. Liu, H. Guo, L. Fu, S. Kaiser, H. Schweizer, and H. Giessen, "Three-dimensional photonic metamaterials at optical frequencies," Nat. Mater., vol. 7, no. 1, pp. 31-37, 2008.

[128] N. Liu, T. Weiss, M. Mesch, et al., "Planar metamaterial analogue of electromagnetically induced transparency for plasmonic sensing," Nano Lett., vol. 10, no. 4, pp. 1103-1107, 2010.

[129] G. Adamo, J. Y. Ou, J. K. So, et al., "Electron-beam-driven collective-mode metamaterial light source," Phys. Rev. Lett., vol. 109, no. 21, p. 217401, 2012.

[130] G. Adamo, K. F. MacDonald, Y. H. Fu, et al., "Light well: a tunable free-electron light source on a chip,” Phys. Rev. Lett., vol. 103, no. 11, p. 113901, 2009.

[131] G. Adamo, K. F. MacDonald, Y. H. Fu, D. P. Tsai, F. G. de Abajo, and N. I. Zheludev, "Tuneable electron-beam-driven nanoscale light source,” J. Opt., vol. 12, no. 2, p. 024012, 2010.

[132] N. I. Zheludev and Y. S. Kivshar, "From metamaterials to metadevices," Nat. Mater., vol. 11, no. 11, pp. 917-924, 2012.

[133] M. V. Gorkunov and M. A. Osipov, "Tunability of wire-grid metamaterial immersed into nematic liquid crystal," J. Appl. Phys., p. 036101, 2008, https://doi.org/10.1063/1.2837099.

[134] J. Y. Ou, E. Plum, L. Jiang, and N. I. Zheludev, "Reconfigurable photonic metamaterials," Nano Lett., vol. 11, no. 5, pp. 21422144, 2011.

[135] Y. Kivshar and N. Zheludev, "From metamaterials to metadevices,” Nat. Mater., vol. 11, no. 7, pp. 197-124, 1879.

[136] L. Ferrari, C. Wu, D. Lepage, X. Zhang, and Z. Liu, "Hyperbolic metamaterials and their applications," Prog. Quant. Electron., vol. 40, pp. 1-40, 2015.

[137] A. Poddubny, I. Iorsh, P. Belov, and Y. Kivshar, "Hyperbolic metamaterials," Nat. Photonics, vol. 7, no. 12, p. 948, 2013.

[138] J. Sun, N. M. Litchinitser, and J. Zhou, "Indefinite by nature: from ultraviolet to terahertz," ACS Photonics, vol. 1, no. 4, pp. 293303, 2014.

[130] N. Talebi, C. Ozsoy-Keskinbora, H. M. Benia, K. Kern, C. T. Koch, and P. A. Van Aken, "Wedge Dyakonov waves and Dyakonov plasmons in topological insulator $\mathrm{Bi}_{2} \mathrm{Se}_{3}$ probed by electron beams," ACS Nano, vol. 10, no. 7, pp. 6988-6994, 2016.

[140] H. L. Andrews, C. H. Boulware, C. A. Brau, and J. D. Jarvis, "Superradiant emission of Smith-Purcell radiation," Phys. Rev. Spec. Top. Accel. Beams, vol. 8, no. 11, p. 110702, 2005.
[141] H. L. Andrews and C. A. Brau, "Gain of a Smith-Purcell freeelectron laser," Phys. Rev. Spec. Top. Accel. Beams, vol. 7, no. 7, p. $070701,2004$.

[142] H. L. Andrews, C. H. Boulware, C. A. Brau, and J. D. Jarvis, "Dispersion and attenuation in a Smith-Purcell free electron laser," Phys. Rev. Spec. Top. Accel. Beams, vol. 8, no. 5, p. $050703,2005$.

[143] P. M. Van den Berg, "Smith-Purcell radiation from a point charge moving parallel to a reflection grating," JOSA, vol. 63 , no. 12, pp. 1588-1597, 1973.

[144] J. T. Donohue and J. Gardelle, "Simulation of Smith-Purcell radiation using a particle-in-cell code," Phys. Rev. Spec. Top. Accel. Beams, vol. 8, no. 6, p. 060702, 2005.

[145] D. Li, Z. Yang, K. Imasaki, and G. S. Park, "Particle-in-cell simulation of coherent and superradiant Smith-Purcell radiation," Phys. Rev. Spec. Top. Accel. Beams, vol. 9, no. 4, p. $040701,2006$.

[146] V. Kumar and K. J. Kim, "Analysis of Smith-Purcell free-electron lasers,” Phys. Rev., vol. 73, no. 2, p. 026501, 2006.

[147] D. Li, K. Imasaki, Z. Yang, and G. S. Park, "Three-dimensional simulation of super-radiant Smith-Purcell radiation," Appl. Phys. Lett., vol. 88, no. 20, p. 201501, 2006.

[148] I. Shih, D. B. Chang, J. E. Drummond, et al., "Experimental investigations of Smith-Purcell radiation," JOSA B, vol. 7, no. 3, pp. 351-356, 1990.

[149] K. J. Woods, J. E. Walsh, R. E. Stoner, H. G. Kirk, and R. C. Fernow, "Forward directed Smith-Purcell radiation from relativistic electrons," Phys. Rev. Lett., vol. 74, no. 19, p. 3808, 1995.

[150] L. Schächter and A. Ron, "Smith-Purcell free-electron laser," Phys. Rev., vol. 40, no. 2, p. 876, 1989.

[151] J. F. Lepage and N. McCarthy, "Analysis of the diffractional properties of dual-period apodizing gratings: theoretical and experimental results," Appl. Opt., vol. 43, no. 17, pp. 3504-3512, 2004.

[152] A. P. Hibbins, J. R. Sambles, and C. R. Lawrence, "Excitation of remarkably nondispersive surface plasmons on a nondiffracting, dual-pitch metal grating," Appl. Phys. Lett., vol. 80, no. 13, pp. 2410-2412, 2002.

[153] D. C. Skigin and R. A. Depine, "Resonances on metallic compound transmission gratings with subwavelength wires and slits," Opt. Commun., vol. 262, no. 2, pp. 270-275, 2006.

[154] W. Liu, Y. Li, H. Jiang, Z. Lai, and H. Chen, "Controlling the spectral width in compound waveguide grating structures," Opt. Lett., vol. 38, no. 2, pp. 163-165, 2013.

[155] K. Ishi, Y. Shibata, T. Takahashi, et al., "Observation of coherent Smith-Purcell radiation from short-bunched electrons," Phys. Rev., vol. 51, no. 6, p. 5212, 1995.

[156] J. Urata, M. Goldstein, M. F. Kimmitt, A. Naumov, C. Platt, and J. E. Walsh, "Superradiant smith-purcell emission," Phys. Rev. Lett., vol. 80, no. 3, p. 516, 1998.

[157] D. Gabor, “A new microscopic principle," Nature, vol. 161, no. 4098, pp. 777-778, 1948.

[158] E. N. Leith and J. Upatnieks, "Reconstructed wavefronts and communication theory," JOSA, vol. 52, no. 10, pp. 1123-1130, 1962.

[159] B. R. Brown, and A. W. Lohmann, "Complex spatial filtering with binary masks,” Appl. Opt., vol. 5, no. 6, pp. 967-969, 1966. 
[160] K. Xu, L. W. Snyman, and H. Aharoni, "Si light-emitting device in integrated photonic CMOS ICs,” Opt. Mater., vol. 69, pp. 274-282, 2017.

[161] J. J. Cowan, "Holography with standing surface plasma waves," Opt. Commun., vol. 12, no. 4, pp. 373-378, 1974.

[162] J. Li, S. Kamin, G. Zheng, F. Neubrech, S. Zhang, and N. Liu, "Addressable metasurfaces for dynamic holography and optical information encryption," Sci. Adv., vol. 4, no. 6, p. 6768, 2018.

[163] L. Huang, S. Zhang, and T. Zentgraf, "Metasurface holography: from fundamentals to applications," Nanophotonics, vol. 7, no. 6, pp. 1169-1190, 2018.

[164] N. Yu, P. Genevet, M. A. Kats, et al., “Light propagation with phase discontinuities: generalized laws of reflection and refraction," Science, vol. 334, no. 6054, pp. 333-337, 2011.

[165] X. Ni, N. K. Emani, A. V. Kildishev, A. Boltasseva, and V. M. Shalaev, "Broadband light bending with plasmonic nanoantennas," Science, vol. 335, no. 6067, p. 427, 2012.

[166] L. Huang, X. Chen, H. Mu hlenbernd, et al., "Dispersionless phase discontinuities for controlling light propagation," Nano Lett., vol. 12, no. 11, pp. 5750-5755, 2012.

[167] S. Larouche, Y. J. Tsai, T. Tyler, N. M. Jokerst, and D. R. Smith, "Infrared metamaterial phase holograms," Nat. Mater., vol. 11, no. 5, pp. 450-454, 2012.

[168] N. K. Grady, J. E. Heyes, D. R. Chowdhury, et al., “Terahertz metamaterials for linear polarization conversion and anomalous refraction," Science, vol. 340, no. 6138, pp. 13041307, 2013.

[169] G. Li, B. P. Clarke, J. K. So, K. F. MacDonald, and N. I. Zheludev, “Holographic free-electron light source," Nat. Commun., vol. 7, no. 1, pp. 1-6, 2016.

[170] M. Harwit, "Photon orbital angular momentum in astrophysics," Astrophys. J., vol. 597, no. 2, p. 1266, 2003.

[171] L. Kipp, M. Skibowski, R. L. Johnson, et al., "Sharper images by focusing soft X-rays with photon sieves," Nature, vol. 414, no. 6860, pp. 184-188, 2001.

[172] Q. Cao and J. Jahns, "Focusing analysis of the pinhole photon sieve: individual far-field model,” J. Opt. Soc. Am. A, vol. 19, no. 12, pp. 2387-2393, 2002.

[173] Q. Cao and J. Jahns, "Nonparaxial model for the focusing of highnumerical-aperture photon sieves," J. Opt. Soc. Am. A, vol. 20, no. 6, pp. 1005-1012, 2003.

[174] O. Carnal, M. Sigel, T. Sleator, H. Takuma, and J. Mlynek, "Imaging and focusing of atoms by a Fresnel zone plate," Phys. Rev. Lett., vol. 67, no. 23, p. 3231, 1991.

[175] K. Jefimovs, O. Bunk, F. Pfeiffer, D. Grolimund, J. F. van der Veen, and C. David, "Fabrication of Fresnel zone plates for hard
X-rays," Microelectron. Eng., vol. 84, nos 5-8, pp. 1467-1470, 2007.

[176] C. Xie, X. Zhu, L. Shi, and M. Liu, "Spiral photon sieves apodized by digital prolate spheroidal window for the generation of hard-x-ray vortex," Opt. Lett., vol. 35, no. 11, pp. 1765-1767, 2010.

[177] G. Andersen, and D. Tullson, "Broadband antihole photon sieve telescope,” Appl. Opt., vol. 46, no. 18, pp. 3706-3708, 2007.

[178] R. Liu, F. Li, M. J. Padgett, and D. B. Phillips, "Generalized photon sieves: fine control of complex fields with simple pinhole arrays," Optica, vol. 2, no. 12, pp. 1028-1036, 2015.

[179] K. Huang, H. Liu, F. J. Garcia-Vidal, et al., "Ultrahigh-capacity nonperiodic photon sieves operating in visible light," Nat. Commun., vol. 6, no. 1, pp. 1-7, 2015.

[180] I. A. Walmsley and C. Dorrer, "Characterization of ultrashort electromagnetic pulses," Adv. Opt. Photonics, vol. 1, no. 2, pp. 308-437, 2009.

[181] P. Vasa, W. Wang, R. Pomraenke, et al., "Real-time observation of ultrafast Rabi oscillations between excitons and plasmons in metal nanostructures with J-aggregates," Nat. Photonics, vol. 7, no. 2, p. 128, 2013.

[182] E. M. Kosik, A. S. Radunsky, I. A. Walmsley, and C. Dorrer, "Interferometric technique for measuring broadband ultrashort pulses at the sampling limit," Opt. Lett., vol. 30, no. 3, pp. 326328, 2005.

[183] A. Yurtsever, R. M. van der Veen, and A. H. Zewail, "Subparticle ultrafast spectrum imaging in 4D electron microscopy," Science, vol. 335, no. 6064, pp. 59-64, 2012.

[184] P. A. Midgley and R. E. Dunin-Borkowski, "Electron tomography and holography in materials science," Nat. Mater., vol. 8, no. 4, pp. 271-280, 2009.

[185] N. van Nielen, M. Hentschel, N. Schilder, H. Giessen, A. Polman, and N. Talebi, "Electrons generate self-complementary broadband vortex light beams using chiral photon sieves," Nano Lett., vol. 20, no. 8, pp. 5975-5981, 2020.

[186] C. Shi, D. K. Luu, Q. Yang, et al., "Recent advances in nanorobotic manipulation inside scanning electron microscopes," Microsystems \& Nanoengineering, vol. 2, no. 1, pp. 1-6, 2016.

[187] P. G. Etchegoin, E. C. L. Ru, and M. Meyer, "An analytic model for the optical properties of gold,” J. Chem. Phys., vol. 125, no. 16, p. 164705, 2006.

[188] P. G. Etchegoin, E. C. L. Ru, and M. Meyer, "Erratum: an analytic model for the optical properties of gold," J. Chem. Phys., vol. 127, no. 18, p. 189901, 2007. 